\title{
MULTIVARIABLE HODGE THEORETICAL INVARIANTS OF GERMS OF PLANE CURVES. II
}

\author{
PIERRETTE CASSOU-NOGUÈS AND ANATOLY LIBGOBER
}

\section{INTRODUCTION}

In the first part of this work, [5], we studied Hodge theoretical invariants of local systems of the complements to germs of plane curve singularities. These invariants, called the faces of quasi-adjunction, yield a refinement of the multivariable Alexander polynomial of a link of isolated singularity or, more precisely, the refinement of the characteristic varieties associated with the fundamental group of the complements to the links. They also provide a multivariable generalization of the spectrum of singularity due to Arnold and Steenbrink (in the case of curves).

In the present paper we develop algorithmic methods for calculating these Hodge theoretical invariants in terms of power series which are the defining equations of the germs. Given such a power series we describe a decorated by integers graph with two types of 0-dimensional cells and two types of 1-dimensional cells (cf. below in this introduction). This graph is called the Newton tree (cf. sect.2.3). After dropping distinction between types of edges in the Newton tree one essentially obtains the splice diagram of Eisenbud and Neumann ([8]) for the link of the singularity of $f$. Newton trees earlier were used for the study of quasi-ordinary power series in $[2]$, ideals in $\mathbb{C}[[x, y]]$ in $[6]$ and plane algebraic curves in [4]. Here we also associate with a germ the toroidal (in the sense of [14]) pair $\left(U_{f}, D\right)$ which provides a resolution in the category of toroidal pairs of the pair $(B, C)$, where $C$ is the germ of plane curve and $B$ is a small ball about the singular point of $C$. Our resolution can as well be viewed as a resolution in the category of orbifolds. Unlike previously used smooth resolutions (cf. references in [25]) use of toroidal resolutions allows to encode whole resolution process into the combinatorial data i.e. the Newton tree. Previous attempts to use mildly singular resolutions in this context were made

First author is partially supported by the grants MTM2010-21740-C02-01 and MTM2010-21740-C02-02. The second author was supported by a grant from J.Simon Foundation. 
in [21], [3], [10]. Use of such type of resolutions is also consistent with philosophy used in the minimal model program (cf. [12] and references there and [27]).

One of the main results in this paper describes the polytopes of quasiadjunction in terms of Newton tree (cf.theorem 4.4). Among other things such description allows to get results on the structure of the polytopes of quasi-adjunction and make many explicit calculations. Since the log-canonical threshold is one of the constants of quasi-adjunction (recall that these constants depend of a choice of germ $\phi \in \mathbb{C}_{0,0}^{2}$ and the log-canonical threshold corresponds to the choice $\phi=1 \mathrm{cf}$. [17]), as a consequence we also obtain explicit description of the polytope which is the (multivariable) log-canonical threshold (called here log-canonical wall) and also the part of the toroidal resolution $U_{f}$ which determines it. Using the relation between the faces of quasi-adjunction and Bernstein ideals obtained in [5], here we obtain a polynomial (a product of linear forms) which divides all the polynomials in the Bernstein ideal.

Here is one of the consequences of calculations in this paper. In example 5.1 we consider a sequence of singularities such that the sequence of constants of quasi-adjunction (corresponding to $\phi=y^{2}$ ) is given by $\frac{13+6 q}{18+8 q}$ which is increasing sequence when $q \rightarrow \infty$. The sequence of log-canonical thresholds for this sequence of singularities is (decreasing) sequence $\frac{2+q}{4 q}$. Recall that set of log-canonical thresholds of singularities contains only finite ascending sequences (cf. [24] for a much more general discussion).

Let us describe the content of the paper in more details. In the first section we recall the definition and construction of Newton trees of a germ $f$. The Newton tree is a tree (with additional structure), built from the Newton polygons that appear at each stage of the Newton algorithm. This additional structure consists with splitting 0-dimensional (resp. 1-dimensional) cells of the tree into two types called vertices and arrows (resp. horizontal and vertical). Each vertex in the Newton tree corresponds to a face of the Newton polygon of a polynomial appearing in a step of the Newton algorithm. It is decorated by integers extracted from the data obtained from the linear form vanishing on a face. The Newton tree determines the dual graph of the smooth resolution of the singularity of $f$ obtained after resolving cyclic quotient singularities of our toroidal resolution. More precisely, we have a bijection between the vertices of the Newton tree and the exceptional divisors in the resolution which intersect other exceptional divisors at least three times ("rupture points" of the dual graph). One 
of the results in this section is that the Newton tree is sufficient to compute the polytopes of quasi-adjunction i.e. only the "rupture points" contribute to the calculation of the polytopes of quasi-adjunction (this was observe in [5] already). In the following section, we prove that this condition is also necessary, that means that all the vertices in the Newton tree contribute to a face of a polytope of quasi-adjunction. This is done using induction. Firstly we show that the intersections of the polytopes of quasi-adjunction with $\left\{s_{1}=1\right\}$ are the polytopes of quasi-adjunction of $f_{2}, \cdots, f_{r}$. Then we prove the result for $r=1$. In particular we retrieve the computation of M.Saito of the exponents between 0 and 1 . Recall that the identification of the constants of quasi-adjunction and the spectrum was made in [19]. The result for $r$ branches follows from the result for $r-1$ branches except in the cases where some vertex doesn't appear in hyperplane $\left\{s_{i}=1\right\}$ for any $i$. We have to work out these cases separately $(r=2$ and $r=3)$.

The final section is devoted to the computation of the log canonical wall. Let $f$ be a germ, we define the Newton nest of $f$, the following way. It is a set of vertices of the Newton tree, consisting in all vertices that correspond to faces of the Newton polygon of $f$ in some system of coordinates. It is a connected set of vertices in the Newton tree. We show that the log canonical polytope is exactly given by the Newton nest of $f$. One can compare this with the result of J.Kollar showing the constant which is the log-canonical threshold of a germ depends only on the first characteristic pair. The article ends with discussion of ACC conditions for constants and polytopes of quasi-adjunction and with additional explicit examples.

\section{Newton trees}

2.1. Newton polygons. For a subset $E \subset \mathbb{N}^{2}$, let $\Delta(E)$ denotes the convex hull of the set $E+\mathbb{R}_{+}^{2}=\left\{a+b, a \in E, b \in \mathbb{R}_{+}^{2}\right\}$. The boundary of $\Delta(E)$ is a polygon with a finite number of vertices and edges. A subset $\Delta \subset \mathbb{R}^{2}$ is called a Newton diagram if there exists a set $E \subset \mathbb{N}^{2}$ such that $\Delta=\Delta(E)$. Let $E_{0}=\left\{v_{0}, \cdots, v_{m}\right\}$ be the set of vertices of $\Delta$ and let $v_{i}=\left(\alpha_{i}, \beta_{i}\right) \in \mathbb{N}^{2}$ with ordering such that $\alpha_{i-1}<\alpha_{i}, \beta_{i-1}>\beta_{i}$ for $i=1, \cdots, m$. For $i=1, \cdots, m$ we denote by $S_{i}=\left[v_{i-1}, v_{i}\right]$ and by $l_{S_{i}}$ the line supporting the segment $S_{i}$. The union of compact edges of the boundary of a Newton diagram is called the Newton polygon. In above notation, it is the union of the edges $S_{i}$ and denoted $\mathcal{N}(\Delta)$. The Newton polygon $\mathcal{N}(\Delta)$ is empty iff $\Delta=\left(\alpha_{0}, \beta_{0}\right)+\mathbb{R}_{+}^{2}$. The integer $h(\Delta)=\beta_{0}-\beta_{m}$ is called the height of $\Delta$. 
Let

$$
f(x, y)=\sum_{(\alpha, \beta) \in \mathbb{N}^{2}} c_{\alpha, \beta} x^{\alpha} y^{\beta} \in \mathbb{C}[[x, y]]
$$

The support of $f$ is

$$
\operatorname{Supp} f=\left\{(\alpha, \beta) \in \mathbb{N} \times \mathbb{N} \mid c_{\alpha, \beta} \neq 0\right\} .
$$

We use $\Delta(f)=\Delta(\operatorname{Supp} f)$ and $\mathcal{N}(f)=\mathcal{N}(\Delta(f))$. For a line $l$ in $\mathbb{R}^{2}$, the initial part of $f$ with respect to $l$ is

$$
\operatorname{in}(f, l)=\sum_{(\alpha, \beta) \in l} c_{\alpha, \beta} x^{\alpha} y^{\beta} .
$$

If the line $l$ has equation $p \alpha+q \beta=N$, with $(p, q) \in\left(\mathbb{N}^{*}\right)^{2}$ and $\operatorname{gcd}(p, q)=1$, then $\operatorname{in}(f, l)$ is zero or a monomial or, if $l=l_{S}$ for some segment $S$ of $\mathcal{N}(\Delta)$, of the form

$$
\operatorname{in}(f, l)=x^{a_{l}} y^{b_{l}} F_{S}\left(x^{q}, y^{p}\right),
$$

where $\left(a_{l}, b_{l}\right) \in \mathbb{N}^{2}$ and

$$
F_{S}(x, y)=c \prod_{1 \leq i \leq n}\left(y-\mu_{i} x\right)^{\nu_{i}},
$$

with $c \in \mathbb{C}^{*}, n \in \mathbb{N}^{*}, \mu_{i} \in \mathbb{C}^{*}$ (all different) and $\nu_{i} \in \mathbb{N}^{*}$.

\subsection{Newton algorithm.}

Definition 2.1. (Newton maps) Let $(p, q) \in \mathbb{N}^{2}, \operatorname{gcd}(p, q)=1$ and $\mu \in \mathbb{C}^{*}$. Let $\left(p^{\prime}, q^{\prime}\right) \in \mathbb{N}^{2}$ such that $q q^{\prime}-p p^{\prime}=1$. The map $\Pi_{(p, q, \mu)}$ : $\mathbb{C}_{\left(x_{1}, y_{1}\right)}^{2} \rightarrow \mathbb{C}_{(x, y)}^{2}$ given by $x=\mu^{q^{\prime}} x_{1}^{p}, y=x_{1}^{q}\left(y_{1}+\mu^{p^{\prime}}\right)$ is called Newton map.

If $\mu=0$, the Newton map is the monomial map given by: $x=x_{1}^{p}, y=$ $x_{1}^{q} y_{1}$

We denote by $\Pi_{(p, q, \mu)}^{*}$ the induced homomorphim $\mathbb{C}[[x, y]] \longrightarrow \mathbb{C}\left[\left[x_{1}, y_{1}\right]\right]$. The change $\left(p^{\prime}, q^{\prime}\right) \rightarrow\left(p^{\prime}+i q, q^{\prime}+i p\right)$ results in change of coordinates: $\left(x_{1}, y_{1}\right) \mapsto\left(\mu^{i} x_{1}, \mu^{-i q} y_{1}\right)$ and does not affect results.

In the sequel we will always assume that $p^{\prime}<q$ and $q^{\prime}<p$. This will make procedures canonical.

Lemma 2.2. [6] Let $f(x, y) \in \mathbb{C}[[x, y]], f \neq 0$ and $\Pi_{(p, q, \mu)}^{*}(f)\left(x_{1}, y_{1}\right)=$ $f_{1}\left(x_{1}, y_{1}\right) \in \mathbb{C}\left[\left[x_{1}, y_{1}\right]\right]$.

(1) If there does not exist a face $S$ of $\mathcal{N}(f)$ whose supporting line has equation $p \alpha+q \beta=k$ with $k \in \mathbb{N}$, then

$$
f_{1}\left(x_{1}, y_{1}\right)=x_{1}^{m} u\left(x_{1}, y_{1}\right)
$$

with $m \in \mathbb{N}, u\left(x_{1}, y_{1}\right) \in \mathbb{C}\left[\left[x_{1}, y_{1}\right]\right]$ and $u(0,0) \neq 0$. 
(2) If there exists a face $S$ of $\mathcal{N}(f)$ whose supporting line has equation $p \alpha+q \beta=k_{0}$ for some $k_{0} \in \mathbb{N}$, and if $F_{S}(1, \mu) \neq 0$, then

$$
f_{1}\left(x_{1}, y_{1}\right)=x_{1}^{k_{0}} u\left(x_{1}, y_{1}\right)
$$

with $u\left(x_{1}, y_{1}\right) \in \mathbb{C}\left[\left[x_{1}, y_{1}\right]\right]$ and $u(0,0) \neq 0$.

(3) If there exists a face $S$ of $\mathcal{N}(f)$ whose supporting line has equation $p \alpha+q \beta=k_{0}$ for some $k_{0} \in \mathbb{N}$, and if $F_{S}(1, \mu)=0$, then

$$
f_{1}\left(x_{1}, y_{1}\right)=x_{1}^{k_{0}} g_{1}\left(x_{1}, y_{1}\right)
$$

with $g_{1}\left(x_{1}, y_{1}\right) \in \mathbb{C}\left[\left[x_{1}, y_{1}\right]\right]$ and $g_{1}(0,0)=0, g_{1}\left(0, y_{1}\right) \neq 0$.

For the proof see [6].

From this lemma, we see that there are a finite number of $(p, q, \mu)$ such that $\Pi_{(p, q, \mu)}^{*}(f)$ is not a monomial times a unit in $\mathbb{C}\left[\left[x_{1}, y_{1}\right]\right]$. These triples are given by the equations of the faces of the Newton polygon and the roots of the corresponding face polynomials.

REMARK 2.3. In the first and second case of Lemma 2.2, the Newton polygon of $f_{1}$ is empty. In the third case, the height of the Newton diagram of $f_{1}$ is less than or equal to the multiplicity of $\mu$ as root of $F_{S}(1, X)$.

We say that $f \in \mathbb{C}[[x, y]]$ is in good coordinates if

(1) $\beta_{m} \neq 0$ or

(2) if $\beta_{m}=0$ and

(a) either $l_{S_{m}}$ has equation $p \alpha+q \beta=N$ with $p \neq 1$ or

(b) if $p=1$, and $m \geq 1$ then $F_{S_{m}}$ has at least two factors,

(c) if $p=1$ and $m=1 F_{S_{m}}$ is not of the form

$$
F_{S_{m}}=c\left(y-\mu_{1} x\right)^{\nu_{1}}\left(y-\mu_{2} x\right)^{\nu_{2}}
$$

Lemma 2.4. If $f \in \mathbb{C}[[x, y]]$ is not in good coordinates, there exist changes of variables in $\mathbb{C}[[x, y]]$ in which it is in good coordinates.

The proof can be found in [15]. One has to take in account that our definition of good coordinates corresponds to their definition of quasigood coordinates. The changes of coordinates we use to put $f$ in good coordinates are $x=x, y=y+h(x)$ with $h \in \mathbb{C}[[x]]$.

We say that $f \in \mathbb{C}[[x, y]]$ is in very good coordinates if it is in good coordinates and

(1) $\alpha_{0} \neq 0$ or

(2) if $\alpha_{0}=0$ and

(a) either $l_{S_{1}}$ has equation $p \alpha+q \beta=N$ with $p \neq 1$ or

(b) if $p=1$ and $m \geq 1$ then $F_{S_{1}}$ has at least two factors. 
Let $f \in \mathbb{C}[[x, y]]$ in very good coordinates. Let $\Pi=\Pi_{(p, q, \mu)}$ be a Newton map. We denote by $f_{\Pi}$ the result of $\Pi^{*}(f)$ after a change of variables so that $f_{\Pi}$ is in good coordinates. Let $\Sigma_{n}=\left(\Pi_{1}, \cdots, \Pi_{n}\right)$ where $\Pi_{i}$ is a Newton map for all $i$, we define $f_{\Sigma_{n}}$ by induction: $f_{\Sigma_{1}}=$ $f_{\Pi_{1}}, f_{\Sigma_{i}}=\left(f_{\Sigma_{i-1}}\right)_{\Pi_{i}}$.

Theorem 2.5. Let $f(x, y) \in \mathbb{C}[[x, y]]$, there exists an integer $n_{0}$ such that, for any sequence $\Sigma_{n}=\left(\Pi_{1}, \cdots, \Pi_{n}\right)$ where $\Pi_{i}$ is a Newton map for all $i$, of length at least $n_{0}, f_{\Sigma_{n}}$ is a monomial up to a unit.

Proof. From Lemma 1.1, we first observe that the number of Newton maps $\Pi$, such that $f_{\Pi}$ is not a monomial times a unit is finite, bounded by the sum on all faces $S$ of the number of roots of $F_{S}$. What we have to show is that the number of successive Newton maps we have to perform so that $f_{\Sigma}$ is a monomial up to a unit, is also finite.

We start with $f$ in very good coordinates. In this system of coordinates, we denote by $h$ the height of $\Delta(f)$. We argue by induction on $h$. If $h=0$, then $f$ is a monomial up to a unit, and $n=0$. Consider the case where $h>0$. In that case, $\mathcal{N}(f)$ is not empty. Choose a face of $\mathcal{N}(f), S$, and a root of $F_{S}$ with multiplicity $\nu$. Let $\alpha p+\beta q=N$ be the equation of the supporting line of $S$. Then $f_{1}\left(x_{1}, y_{1}\right)=x_{1}^{N} g_{1}\left(x_{1}, y_{1}\right) \in \mathbb{C}\left[\left[x_{1}, y_{1}\right]\right]$ and the height of $\Delta\left(f_{1}\right)$ is $\nu<h$ since $f$ is in good coordinates.

If $f$ is in very good coordinates, we define the length of the Newton algorithm $\mathcal{A}$ applied to $f, d(f, \mathcal{A})$ by induction. If $f$ is a monomial up to a unit, then $d(f, \mathcal{A})=0$. Otherwise $d(f, \mathcal{A})=\max d\left(f_{\Pi}\right)+1$ where the maximum is taken over all faces $S$ of the Newton polygon and all roots of $F_{S}$. Note that the definition of the length depends on the choice of good coordinates at each step of the Newton algorithm.

2.3. Newton trees. Given $f \in \mathbb{C}[[x, y]]$ in very good coordinates, the Newton algorithm consists in applying successive Newton maps attached to successive Newton polygons and changes of variables until the result is a monomial times a unit.

Newton trees are trees that encode the Newton algorithm. They are build by induction, and defined via gluing certain graphs associated to a Newton diagram. More specifically:

Definition 2.6. An abstract Newton tree is a graph with no loops with two types of 0-dimensional cells, called vertices and arrows and two types of 1-dimensional cells called horizontal and vertical edges.

Decoration of an abstract Newton tree is assignment of an integer to a vertex or arrow (represented below in parenthesis) and assignment an 
integer to each end of an edge. Below the integers assigned to unmarked ends are considered to be equal to 1.

All abstract Newton trees have one marked arrow called upper arrow.

\subsubsection{Graph associated to a Newton diagram.}

Definition 2.7. 1. Graph associated to a Newton diagram is an abstract Newton tree with vertices $a_{1}, a_{2}, \ldots a_{m}$ which are in (ordered) 11-correspondence with compact 1-dimensional faces $S_{i}$ of the boundary of Newton diagram, arrows $a_{0}, a_{m+1}$ corresponding to the non-compact faces of the boundary of Newton diagram and $m+1$ vertical edges connecting $a_{i}$ and $a_{i+1}$ for $i=0, \ldots, m$. Increase of subscript corresponds to the downward moving on the graph. The arrow $a_{0}$ is called the upper arrow.

If $\Delta=\left(\alpha_{0}, \beta_{0}\right)+\mathbb{R}_{+}^{2}$ then the graph of $\Delta$ is defined as follows: the graph has no vertices and it has one edge incident to two arrows and the edge is vertical. The upper arrow is defined as the arrow decorated by $\left(\alpha_{0}\right)$ and the arrow at the bottom is decorated by $\left(\beta_{0}\right)$.

Now let us describe the decoration of the graph of a Newton diagram. If the non compact faces of the Newton diagram are $\alpha=\alpha_{0}$ and $\beta=\beta_{m}$ then the upper arrow is decorated by $\left(\alpha_{0}\right)$ and the arrow at the bottom is decorated by $\beta_{m}$. The edges incident to the arrows are decorated with 1 near the arrows. The extremities of the edges are decorated the following way: A vertex corresponds to a face $S$ whose supporting line has equation $p \alpha+q \beta=N$. We decorate the extremity of the edge above the vertex by $q$, and the extremity of the edge under the vertex by $p$. We decorate the vertex by $(N)$.

Note that one can recover the whole Newton polygon from the graph since we can read the equations of the supporting lines of the faces on the graph i.e. the data given by the graph and by the Newton diagram are identical.

2.3.2. Newton tree of $f \in \mathbb{C}[[x, y]]$. The Newton tree of $f$ is defined by induction on the length. Suppose that $f$ has length 0 . Then $f$ is a monomial times a unit and we define its Newton tree as the graph of its Newton diagram (cf. def. 2.7).

Assume that we have constructed the Newton tree for all $f$ of length less or equal to $n-1$. Let $f \in \mathbb{C}[[x, y]]$ in very good coordinates and having length $n$. We define the Newton tree of $f$ in terms of the following data. On one hand the definition 2.7 provides the graph associated to its Newton diagram. On the other hand, for each edge of the Newton polygon and each root of the polynomial corresponding to this edge via the Newton map $\Pi$ and subsequent change to good 


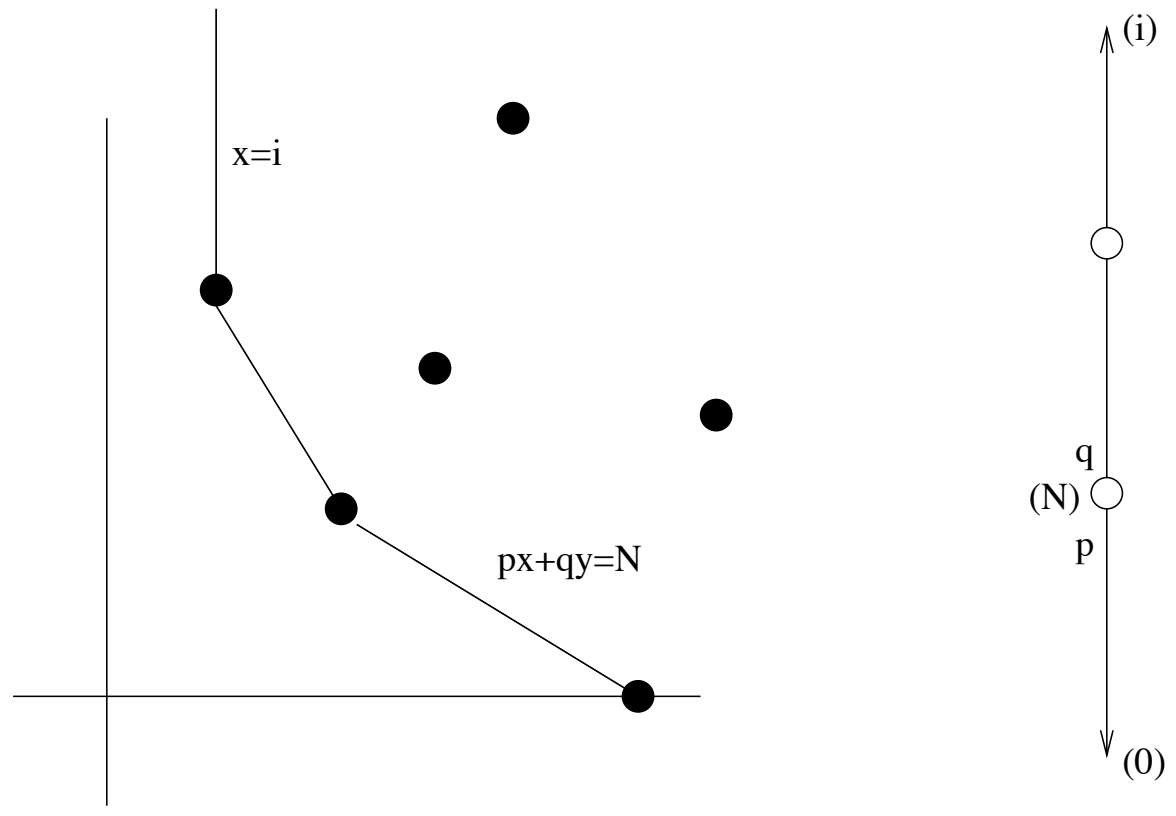

Figure 1

coordinates we obtain the polynomial $f_{\Pi}$ of length at most $n-1$. The assumption of induction yields the Newton tree of $f_{\Pi}$ (for each edge of the Newton polygon of $f$ ).

Definition 2.8. The Newton tree of $f$ is the tree obtained from the above data as follows. Delete the upper arrow of the Newton tree of each $f_{\Pi}$ (recall that each $\Pi$ corresponds to a vertex of the graph of Newton diagram of $f$ ) and glue the edge which was incident to that arrow to the corresponding vertex on the graph of the Newton diagram of $f$ for all П. Moreover, the edges that are glued are renamed to horizontal edges. All other edges of the graph of the Newton diagram of $f$ and the Newton trees of $f_{\Pi}$ retain the labels which they had as edges of the trees. The upper arrow of the graph of the Newton diagram of $f$ is declared the upper arrow of the Newton tree of $f$.

To sum up, in this construction the vertices on graph of $f$ are all incident to vertical edges and correspond to the faces on the Newton polygon of some $f_{\Pi}$. The horizontal edges correspond to the successive Newton maps used to construct polynomials $f_{\Pi}$.

Decorations of the Newton tree of $f$ are defined in terms of decorations of the graph of Newton diagram of $f$ and the decorations of $f_{\Pi}$ as follows. 
Definition 2.9. Let $v$ be a vertex of a Newton tree. If $v$ corresponds to a face of the Newton polygon of $f$, we say that $v$ has no preceding vertex and we define $\mathcal{S}(v)=\{v\}$. Let $v$ be a vertex on a Newton tree. It is on the Newton polygon of $f_{\Sigma}$. The Newton tree of $f_{\Sigma}$ has been glued on a vertex $v_{1}$ which is called the preceding vertex of $v$. If $v_{1}$ does not correspond to a face of the Newton polygon of $f$, we can consider its preceding vertex $v_{2}$. Then we can define $\mathcal{S}(v)=\left\{v_{i}, \cdots, v_{2}, v_{1}, v\right\}$, where $v_{i}$ has no preceding vertex and $v_{j}$ is the preceding vertex of $v_{j-1}$ for $2 \leq j \leq i$.

The final Newton tree is decorated the following way. The decorations of the arrows and vertices are not changed. The decorations of the edges are changed. Let $v$ be a vertex on the Newton tree. If $\mathcal{S}(v)=\{v\}$ then the decorations near $v$ are not changed. If $\mathcal{S}(v)=\left\{v_{i}, \cdots, v_{2}, v_{1}, v\right\}$ and if the decoration near $v$ on the Newton tree such that $\mathcal{S}(v)=\left\{v_{i-1}, \cdots, v_{2}, v_{1}, v\right\}$ (that is on the Newton tree which is glued at $\left.v_{i}\right)$, are $(m, p)$, after the gluing, the decorations near $v$ are $\left(m+p_{i} q_{i} p_{i-1}^{2} \cdots p_{1}^{2} p, p\right)$.

As an example the following is the Newton tree of $f(x, y)=\left(x^{2}-\right.$ $\left.y^{3}\right)^{2}\left(x^{3}-y^{2}\right)^{2}+x^{6} y^{3}+x^{5} y^{5}+x^{4} y^{7}$
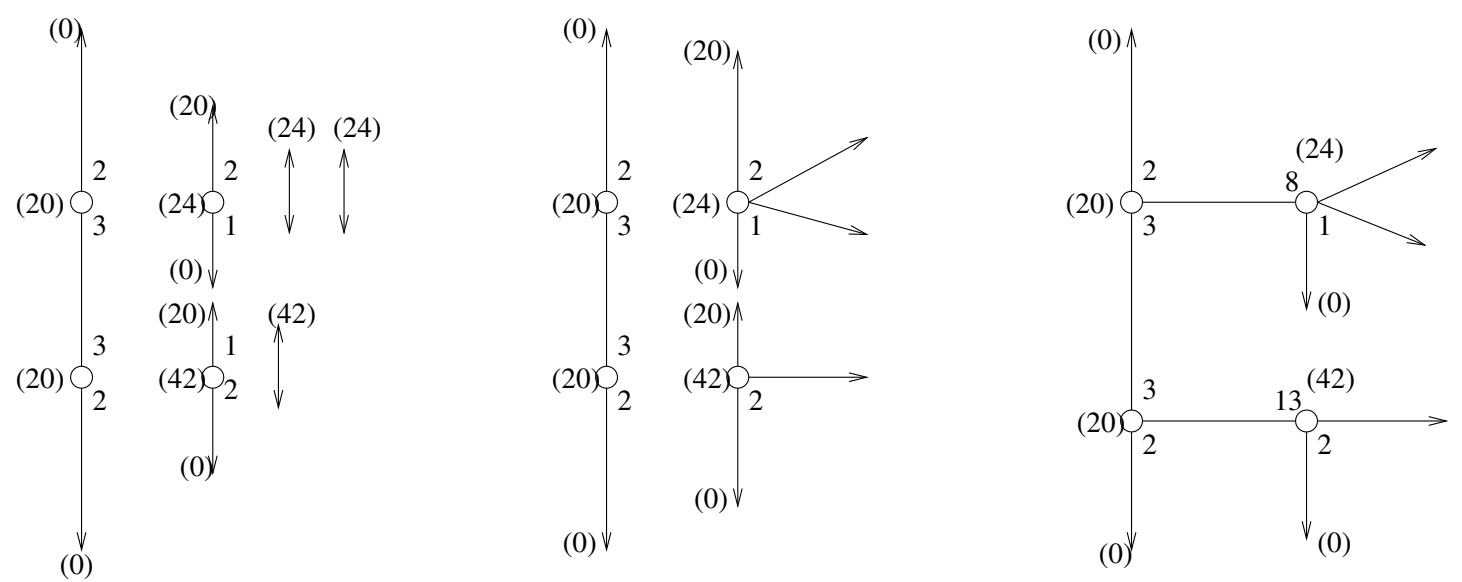

\section{FiguRe 2}

If we add an arrow to a vertex of a Newton tree, this arrow defines a germ of curve. This germ of curve is called a curvette of the vertex. If $v$ is the vertex, we denote by $\mathcal{C}_{v}$ its curvette. It is called a virtual component in [8].

Note that on the edges arising for a vertex there are at most two decorations on the ends near the vertex which are different from 1 . We call them nearby decorations of the vertex. 
2.4. Change of variables. Newton trees are constructed using a very good system of coordinates. But very good systems of coordinates are not unique. We want to study the Newton trees in different systems of very good coordinates.

Consider a system of very good coordinates for $f$. Consider the Newton polygon of $f$ in this system of coordinates.

If there is no face of the Newton polygon with equation $p \alpha+q \beta=N$ with $p$ or $q$ equal to 1 , then there is no other system of good coordinates.

If there is a face of the Newton polygon with equation $p \alpha+q \beta=N$ with $p$ or $q$ equal to 1 , assume $p=1$. Let

$$
c x^{a_{S}} y^{b_{S}} \prod^{k_{S}}\left(y-\mu_{i} x^{q}\right)^{\nu_{i}}
$$

be the face polynomial. Since we are in very good coordinates, we have $b_{S} \neq 0$ or if $q \neq 1, k_{S}>1$, and if $q=1, k_{S}>2$.

We make the change of variables $x=x, y=y+a x^{q}$.

The faces above $S$ do not change neither their face polynomial. The face $S$ has the same supporting line but its face polynomial is now

$$
c x^{a_{S}}\left(y-a x^{q}\right)^{b_{S}} \prod^{k_{S}}\left(y-\left(\mu_{i}-a\right) x^{q}\right)^{\nu_{i}}
$$

If $a=\mu_{i}$, for some $i$, the face doesn't hit the $x$-axis. If $a \neq \mu_{i}$ for all $i$, the face hits the $x$-axis, but anyway we are still in very good coordinates.

We want to compare the Newton trees in these two systems of coordinates.

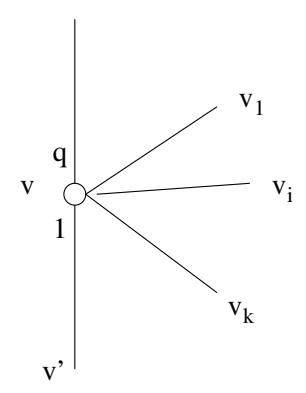

\section{FIGURE 3}

We consider the vertex $v$ which represents the face $S$. There are $k_{S}$ horizontal edges starting from $v$ corresponding to each root $\mu$ of the face polynomial. There is a vertical edge decorated with 1 near $v$ under $v$ and a vertical edge decorated with $q$ above $v$. All the edges can be ended by vertices or arrows. We denote these ends by $v_{i}$ for the 
horizontal edges and $v^{\prime}$ for the vertical edge pointing downward. We make the change of variables $x=x, y=y+a x^{q}$.

(1) If $a \neq \mu_{i}$ for all $i$. Then the new Newton tree is in Figure 4. We have $k_{S}+1$ horizontal edges, one for each of the roots $\mu_{i}$ and one for $a$. We proved in [7] that in this case, we cut out the Newton tree in two pieces on the vertical edge under $v$. We have $\mathcal{T}_{a}$ which contains $v$ and $\mathcal{T}_{u}$ which contains the vertical edge ending with $v^{\prime}$. We stick back $\mathcal{T}_{u}$ on $v$ making the vertical edge ending with $v^{\prime}$, horizontal and we add a vertical edge decorated with 1 ending with an arrow decorated with (0) (On Figure 6, we start with Newton tree 2 or 3 and get Newton tree 1).

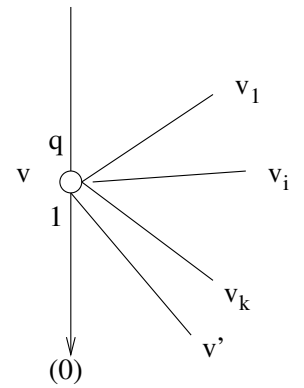

FiguRe 4

(2) If $a=\mu_{i}$, We cut the Newton tree in 3 pieces. We cut the vertical edge ending with $v^{\prime}$, we cut the horizontal edge ending with $v_{i}$. We have the piece containing $v$, the piece containing $v^{\prime}$ and the piece containing $v_{i}$. We stick the piece containing $v_{i}$ making the edge ending with $v_{i}$ vertical. We stick the piece containing $v^{\prime}$ on $v$ making the edge containing $v^{\prime}$ horizontal. We call this operation exchange of vertical edge (On Figure 6, we exchange Newton trees 2 and 3).

Note that some faces may appear on the Newton polygon in some system of very good coordinates, but that there is not always a system of coordinates such that they all appear at the same time.

EXAMPLE 2.10. :

In this example, the 3 vertices can correspond to faces of a Newton polygon in some system of coordinates, but at most two of them appear in the same system of coordinates. 


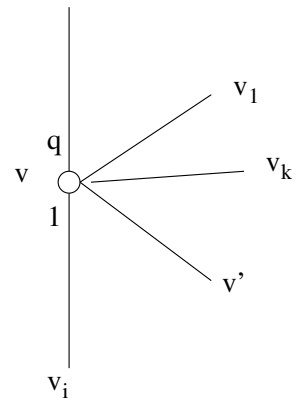

\section{FiguRE 5}
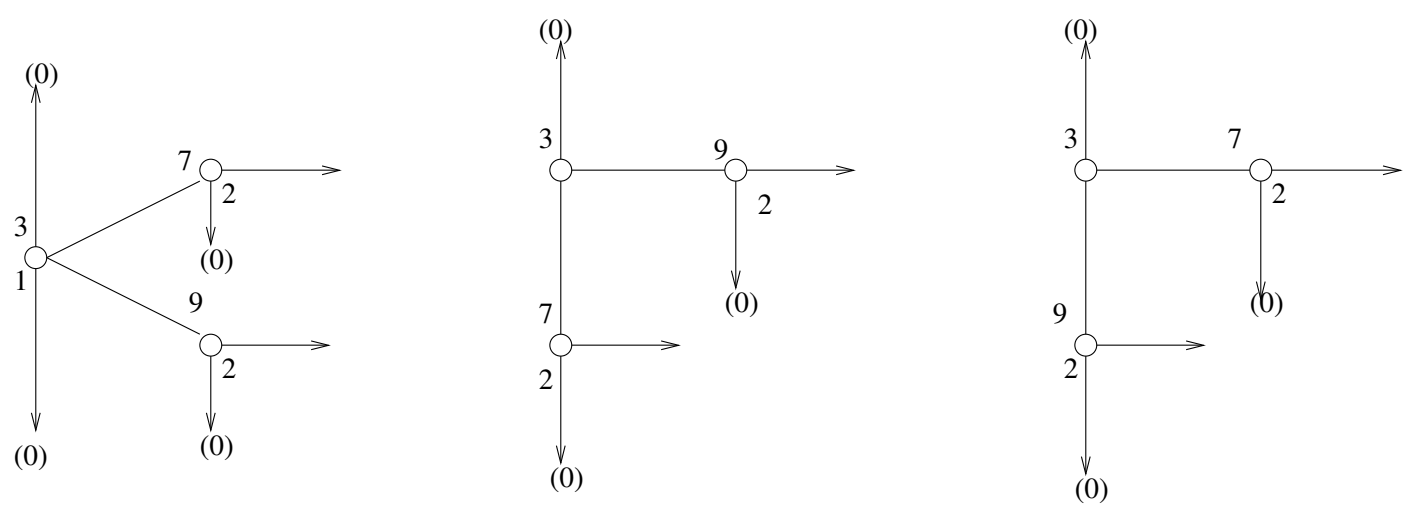

\section{FigURE 6}

Definition 2.11. We say that two Newton trees are equivalent if they differ by exchanging vertical and horizontal edges, and eventually deleting horizontal edges ending with arrows decorated with (0).

We can chose a canonical representant of an equivalent class of Newton tree the following way: At each vertex the only possible edge decorated with 1 near the vertex which is not horizontal ends with an arrow decorated with $(0)$.

In Figure 6, the three Newton trees are equivalent. The canonical representant of the class is the first one.

Equivalent Newton trees represent $f$ in different systems of good coordinates.

Proposition 2.12. Newton trees of $f \in \mathbb{C}[[x, y]]$ in different systems of good coordinates have the same number of vertices. This number is called the Newton complexity of $f$.

\subsection{Combinatorial properties of Newton trees. .}


Proposition 2.13. If $v_{0}$ is the preceding vertex of $v$ with nearby decorations respectively $\left(q_{0}, p_{0}\right)$ and $(q, p)$, we have

$$
q=p_{0} q_{0} p+\tilde{m} .
$$

where $(\tilde{m}, p)$ are the nearby decorations of $v$ on the Newton tree where $\mathcal{S}(v)=\{v\}$.

Proof. See [6].

Definition 2.14. Consider a path on a Newton tree. We say that a number is adjacent to this path if it is not on the path and is a nearby decoration of a vertex on the path. If the path contains an arrow, the decoration of the arrow is a number adjacent to the path.

Definition 2.15. Consider an edge on a Newton tree, its edge determinant is the difference between the product of the numbers on the edge and the product of the numbers adjacent to the edge when the edge is incident to two vertices. If the edge is incident to one arrow, its edge determinant is the product of the decorations on the edge.

Corollary 2.16. (1) In the process of gluing, the edge determinants remain constant.

(2) All edges determinants are strictly positive integers.

Proof. This is a consequence of the previous proposition.

Proposition 2.17. The decoration $(N)$ of a vertex $v$ on a Newton tree is the sum over all the arrows $\mathcal{F}$ of the tree, of the products of the numbers adjacent to the paths $[v, \mathcal{F}]$.

For a proof see [6], Proposition 3.3.

REMARK 2.18. From this proposition we see that when the tree is constructed, the decorations of the vertices are not needed any more because we can compute them from the decorations of the edges. But, anyway, we have to keep in mind that we know them from the beginning.

\subsection{Computation of the intersection multiplicity using New- ton trees.}

Proposition 2.19. The intersection multiplicity of two branches $f$ and $g$ is equal to the product of all the numbers adjacent to the path going from the arrow representing $f$ to the arrow representing $g$ on any Newton tree where $f$ and $g$ are represented.

Corollary 2.20. The decoration $N_{v}$ of a vertex $v$ of a Newton tree of a germ $f$ is equal to the intersection multiplicity of the curvette $\mathcal{C}_{v}$ and $f$. 
See [6] Proposition 5.3.

Given a Newton tree, the arrows decorated with positive multiplicities correspond to branches with the same multiplicity. Along horizontal paths from the first vertical line to any arrow one can compute the Puiseux pairs of the branch. One can also compute the intersection multiplicity of any two branches. Then the data of the Newton tree of $f$ give the topological type of $f$. Given a decorated tree satisfying the condition of positivity of edge determinants there exist germs $f$ with this Newton tree.

The Newton tree of $f$, without specification of edges as horizontal or vertical coincide with the splice diagram of the link of the singularity of $f$ at the origin defined by Eisenbud and Neumann [8].

\section{NeWton space}

In this section for a series $f \in \mathbb{C}[[x, y]]$, we describe a morphism $\pi_{f}: U_{f} \rightarrow \mathbb{C}^{2}$, where $U_{f}$ is a toroidal variety with quotient singularites, $\pi_{f}$ is birational and has the property that the proper preimage of $f=0$ does not intersect the singular locus of $U_{f}$ and is transversal to the exceptional set of $\pi_{f}$. Its construction is much simpler than the construction of log-resolution of pair $\left(\mathbb{C}^{2}, C\right)$ where $C$ is the zero set of $f$. We show that $U_{f}$ comes with the atlas of affine surfaces which are global quotients by cyclic group and hence provides resolution of pair $\left(\mathbb{C}^{2}, C\right)$ in the category of orbifolds.

3.1. Factorization of monomial maps. Let $N$ be a free abelian group of rank two with fixed basis $\left\{E_{1}, E_{2}\right\}$. We use the latter to identify $N$ with $\mathbb{Z}^{2}$. Elements of $N$ will be represented as column vectors e.g. $E_{1}$ and $E_{2}$ correspond to ${ }^{t}(0,1)$ and ${ }^{t}(1,0)$ respectively. Let $N^{+}$be the subset of vectors with positive coordinates.

3.1.1. The variety $U_{\sigma}$. Let $\left\{P_{1}, P_{2}\right\}$ be a pair of primitive vectors in $N^{+}$. Let $P_{i}={ }^{t}\left(p_{i}, q_{i}\right)$ and assume that $\Delta=\operatorname{det}\left(P_{1}, P_{2}\right)=p_{2} q_{1}-p_{1} q_{2}>$ 0 . Let

$$
\sigma=\operatorname{cone}\left(P_{1}, P_{2}\right)=\left\{t P_{1}+s P_{2}, t, s \geq 0\right\}
$$

The toric surface corresponding to this cone will be denoted $U_{\sigma}$ (cf. [9], p. 4). It is biregular to a quotient of $\mathbb{C}^{2}$ by a cyclic group. Let us recall the description of the order of this cyclic group and its action yielding $U_{\sigma}$.

Let ${ }^{t}\left(p_{1}^{\prime}, q_{1}^{\prime}\right)$ be the unique vector such that $p_{1}^{\prime} q_{1}-p_{1} q_{1}^{\prime}=1$ and $0 \leq p_{2} q_{1}^{\prime}-q_{2} p_{1}^{\prime}<p_{2} q_{1}-p_{1} q_{2}$. The relation

$$
{ }^{t}\left(p_{2}, q_{2}\right)=c^{t}\left(p_{1}, q_{1}\right)+d^{t}\left(p_{1}^{\prime}-p_{1}, q_{1}^{\prime}-q_{1}\right)
$$


yields two integers $c, d$ and we have

$$
d=\Delta, \quad c=\Delta-\left(p_{2} q_{1}^{\prime}-q_{2} p_{1}^{\prime}\right)>0, \quad \operatorname{gcd}(c, d)=1
$$

Let $G_{d}$ be the group of $d$-roots of unity. Then the variety $U_{\sigma}$ can be parametrized via

$$
\begin{aligned}
& \mathbb{C}^{2} \quad \longrightarrow \quad \mathbb{C}^{2} / G_{d} \quad \simeq \quad U_{\sigma} \\
& \left(t_{1}, t_{2}\right) \mapsto\left(\zeta_{d}^{-c} t_{1}, \zeta_{d} t_{2}\right) \mapsto\left(u_{1}=t_{1} t_{2}^{c}, u_{2}=t_{1}^{d}, u_{3}=t_{2}^{d}\right)
\end{aligned}
$$

We can also define the variety $U_{\sigma}$ the following way. Let ${ }^{t}\left(p_{2}^{\prime \prime}, q_{2}^{\prime \prime}\right)$ be the unique vector such that $p_{2} q_{2}^{\prime \prime}-p_{2}^{\prime \prime} q_{2}=1$ and $p_{2}^{\prime \prime} q_{1}-q_{2}^{\prime \prime} p_{1}<p_{2} q_{1}-q_{2} p_{1}$. We can write

$$
{ }^{t}\left(p_{1}, q_{1}\right)=\tilde{c}^{t}\left(p_{2}, q_{2}\right)+d^{t}\left(p_{2}^{\prime \prime}-p_{2}, q_{2}^{\prime \prime}-q_{2}\right),
$$

where $\tilde{c}=\Delta-\left(p_{2}^{\prime \prime} q_{1}-q_{2}^{\prime \prime} p_{1}\right)>0$.

$$
\begin{aligned}
& \mathbb{C}^{2} \quad \longrightarrow \quad \mathbb{C}^{2} / G_{d} \simeq \quad U_{\sigma} \\
& \left(t_{1}, t_{2}\right) \mapsto\left(\zeta_{d} t_{1}, \zeta_{d}^{-\tilde{c}} t_{2}\right) \mapsto\left(\tilde{u}_{1}=t_{2}^{d}, \tilde{u}_{2}=t_{1}^{\tilde{c}} t_{2}, \tilde{u}_{3}=t_{1}^{d}\right)
\end{aligned}
$$

Note that $c \tilde{c}$ is congruent to 1 modulo $d$.

Definition 3.1. Let $\Pi_{\sigma}: \mathbb{C}^{2} \rightarrow \mathbb{C}^{2}$ be given by:

$$
\left(t_{1}, t_{2}\right) \mapsto\left(x=t_{1}^{p_{1}} t_{2}^{p_{2}}, y=t_{1}^{q_{1}} t_{2}^{q_{2}}\right)
$$

The maps $\kappa_{\sigma}$ and $\pi_{\sigma}$ in the diagram:

$$
\mathbb{C}^{2} \stackrel{\kappa_{\sigma}}{\longrightarrow} U_{\sigma} \stackrel{\pi_{\sigma}}{\longrightarrow} \mathbb{C}^{2}
$$

will be called the (toric) uniformization and the (toric) blow up respectively.

Lemma 3.2. The morphism $\pi_{\sigma}: U_{\sigma} \rightarrow \mathbb{C}^{2}$ is birational.

Proof. Let $(x, y)$ be the coordinates on $\mathbb{C}^{2}$. We have

$$
u_{1}=\frac{y^{p_{1}^{\prime}-p_{1}}}{x^{q_{1}^{\prime}-q_{1}}}, \quad u_{2}=\frac{y^{p_{2}}}{x^{q_{2}}}, \quad u_{3}=\frac{x^{q_{1}}}{y^{p_{1}}}
$$

The lines $D_{3}:=\left\{u_{1}=0, u_{2}=0\right\}$ and $D_{2}:=\left\{u_{1}=0, u_{3}=0\right\}$ are contained in $U_{\sigma}$. The line $D_{3}$ contracts by $\pi_{\sigma}$ on the origin in $\mathbb{C}^{2}$ if and only $p_{1}$ is different from 0 , and the line $D_{2}$ contracts by $\pi_{\sigma}$ on the origin in $\mathbb{C}^{2}$ if and only if $q_{2}$ is different from 0 . If $d>1$ the origin in $\mathbb{C}^{3}$ is a singular point in $U_{\sigma}$ with a quotient singularity. 
3.1.2. Gluing $U_{\sigma}$ and $U_{\sigma^{\prime}}$. Let $\sigma$ be the cone cone $\left(P_{1}, P_{2}\right)$ as before and $\sigma^{\prime}$ be the cone $\left(P_{2}, P_{3}\right)$ with $P_{3}=^{t}\left(p_{3}, q_{3}\right)$ and $p_{3} q_{2}-p_{2} q_{3}>0$.

We have

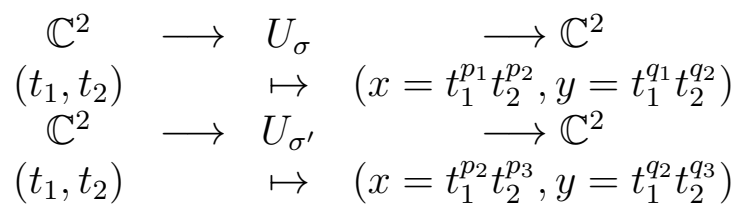

We glue $U_{\sigma}$ and $U_{\sigma^{\prime}}$ along $D_{2}$ on $U_{\sigma}$ and $D_{3}^{\prime}$ on $U_{\sigma^{\prime}}$. We have $u_{2}=\frac{y^{p_{2}}}{x^{q_{2}}}$ and $u_{3}^{\prime}=\frac{x^{q_{2}}}{y^{p_{2}}}$. Then let $A$ be a point on $D_{2}$. Its coordinates are $(0, \xi, 0)$ on $U_{\sigma},(0,0, \xi)$ on $U_{\sigma^{\prime}}$ and $\pi_{\sigma}(A)=\pi_{\sigma^{\prime}}(A)$.

3.1.3. Decomposition of $\sigma$ into $\sigma_{1} \cup \sigma_{1}^{\prime}$. Let $P_{3}=\left(p_{3}, q_{3}\right)$ be such that $p_{3} q_{1}-q_{3} p_{1}>0$ and $p_{2} q_{3}-p_{3} q_{2}>0$. Denote by $\sigma_{1}$ the cone cone $\left(P_{1}, P_{3}\right)$. Let $\left(u_{1}, u_{2}, u_{3}\right)$ the coordinates in $\mathbb{C}^{3}$ such that $U_{\sigma} \subset \mathbb{C}_{u_{1}, u_{2}, u_{3}}^{3}$ and $\left(u_{1}^{1}, u_{2}^{1}, u_{3}^{1}\right)$ the coordinates in $\mathbb{C}^{3}$ such that $U_{\sigma_{1}} \subset \mathbb{C}_{u_{1}^{1}, u_{2}^{1}, u_{3}^{1}}^{3}$. Let $\left(c_{1}, d_{1}\right)$ be defined as before for the cone $\sigma_{1}$.

We have by $\pi_{\sigma}^{-1}$

$$
\begin{gathered}
\mathbb{C}^{2} \quad \longrightarrow \\
(x, y) \quad \mapsto \quad\left(u_{1}=\frac{y^{p_{1}^{\prime}-p_{1}}}{x^{q_{1}^{\prime}-q_{1}}}, \quad u_{2}=\frac{y^{p_{2}}}{x^{q_{2}}}, \quad u_{3}=\frac{x^{q_{1}}}{y^{p_{1}}}\right)
\end{gathered}
$$

The morphism $\pi_{\sigma_{1}}$ is the factorization to $U_{\sigma_{1}}$ of the morphism

$$
\begin{aligned}
& \mathbb{C}^{2} \quad \longrightarrow U_{\sigma_{1}} \quad \longrightarrow \mathbb{C}^{2} \\
& \left(v_{1}, v_{2}\right) \quad \mapsto \quad\left(x=v_{1}^{p_{1}} v_{2}^{p_{3}}, y=v_{1}^{q_{1}} v_{2}^{q_{3}}\right)
\end{aligned}
$$

After a short computation, we deduce

$$
u_{1}=v_{1} v_{2}^{c_{1}}=u_{1}^{1}, u_{2}=v_{1}^{q_{1} p_{2}-q_{2} p_{1}} v_{2}^{q_{3} p_{2}-p_{3} q_{2}}, u_{3}=v_{2}^{d_{1}}=u_{3}^{1}
$$

Then we have a morphism from $\mathbb{C}^{2}$ to $U_{\sigma}$ which factorizes to $U_{\sigma_{1}}$. Denote by $\pi_{\sigma_{1}, \sigma}$ this factorization. We have

$$
\pi_{\sigma_{1}}=\pi_{\sigma} \circ \pi_{\sigma_{1}, \sigma}
$$

The morphism $\pi_{\sigma_{1}, \sigma}$ is the identity from $D_{3}^{1}$ on $D_{3}$ and the line $D_{2}^{1}$ contracts to the singular point in $U_{\sigma}$.

We can also consider the cone $\sigma_{1}^{\prime}$, cone $\left(P_{3}, P_{2}\right)$. Using the second parametrization of $U_{\sigma}$ we can define the same way as before, a morphism $\pi_{\sigma_{1}^{\prime}, \sigma}$. We have

$$
\pi_{\sigma_{1}^{\prime}}=\pi_{\sigma} \circ \pi_{\sigma_{1}^{\prime}, \sigma}
$$

Now the morphism $\pi_{\sigma_{1}^{\prime}, \sigma}$ is the identity from $D_{2}^{1^{\prime}}$ on $D_{2}$ and $D_{3}^{1^{\prime}}$ contracts on the singularity of $U_{\sigma}$. We can glue $U_{\sigma_{1}}$ and $U_{\sigma_{1}^{\prime}}$ as before and we have a morphism from this new variety to $U_{\sigma}$. 
3.1.4. Newton maps. Let $\left\{P_{1}, \cdots, P_{m}\right\}$ be given positive primitive integral vectors in $N^{+}$. We denote by $P_{0}=E_{1}$ and $P_{m+1}=E_{2}$.

To begin, we consider the cone $\sigma_{m}=\operatorname{cone}\left(P_{0}, P_{m}\right)$ and the cone $\sigma_{m}^{\prime}=\operatorname{cone}\left(P_{m}, P_{m+1}\right)$. We consider the variety $U_{\sigma_{m}}$ and the morphism $\pi_{\sigma_{m}}$ which is the factorization of the Newton map

$$
\begin{array}{ccc}
\mathbb{C}^{2} & \longrightarrow & \underset{\sigma_{m}}{U_{1}} \\
\left(t_{1}, t_{2}\right) & \mapsto \mathbb{C}^{2} & \mapsto\left(x=t_{2}^{p_{m}}, y=t_{1} t_{2}^{q_{m}}\right)
\end{array}
$$

We glue $U_{\sigma_{m}}$ and $U_{\sigma_{m}^{\prime}}$ along $D_{2}^{m}$ and $D_{3}^{\prime m}$. We get a variety $U_{m}$ and a birational morphism $\pi_{m}$ which is $\pi_{\sigma_{m}}$ on the chart $U_{\sigma_{m}}$. The line $D_{2}^{m}=D_{3}^{\prime m}$ contracts on the origin of $\mathbb{C}^{2}$. The lines $D_{3}^{m}$ and $D_{2}^{\prime m}$ are not contracted. There is a singularity at the origin of $U_{\sigma_{m}}$ (resp. $U_{\sigma_{m}^{\prime}}$ ) if and only if the cone $\sigma_{m}$ (resp. $\sigma_{m}^{\prime}$ ) is not regular.

Next we consider the subdivision of $\sigma_{m}$ in two cones $\sigma_{m-1}=\operatorname{cone}\left(P_{0}, P_{m-1}\right)$ and the cone $\sigma_{m-1}^{\prime}=\operatorname{cone}\left(P_{m-1}, P_{m}\right)$. We consider the variety $U_{\sigma_{m-1}}$ and the map $\pi_{\sigma_{m-1}}$ which is the factorization of the Newton map

$$
\begin{array}{ccc}
\mathbb{C}^{2} & \longrightarrow & U_{\sigma_{m-1}} \\
\left(t_{1}, t_{2}\right) & \stackrel{\mathbb{C}^{2}}{\mapsto} & \left(x=t_{2}^{p_{m-1}}, y=t_{1} t_{2}^{q_{m-1}}\right)
\end{array}
$$

This map factorizes through $U_{\sigma_{m}}$. When we glue $U_{\sigma_{m-1}}$ and $U_{\sigma_{m-1}^{\prime}}$ we have a birational morphism $\pi_{m, m-1}$ from this variety on $U_{\sigma_{m}}$ which is the identity on $D_{2}^{\prime m-1}$. We glue $U_{\sigma_{m}^{\prime}}$ along $D_{3}^{\prime m}$ and extend $\pi_{m, m-1}$ by the identity in this chart. We obtain a variety $U_{m-1}$ and a birational map $\pi_{m, m-1}$ from $U_{m-1}$ to $U_{m}$. On the chart $U_{\sigma_{m-1}}$ we have

$$
\pi_{\sigma_{m}} \circ \pi_{m, m-1}=\pi_{\sigma_{m-1}}
$$

Finally we get a toric variety $U$ associated to the subdivision and a birational morphism $\pi$ from $U$ to $\mathbb{C}^{2}$. Along one exceptional divisor $E$, the morphism $\pi$ is the Newton map $\pi_{\sigma_{i}}$ where $E=D_{2}^{i}$ on $U_{\sigma_{i}}$. The variety $U$ is smooth if and only if the subdivision is regular.

We denote $\pi^{-1}(0)=\cup_{i=1}^{i=m} E\left(P_{i}\right)$ where $E\left(P_{1}\right)=D_{2}^{1}, E\left(P_{2}\right)=D_{2}^{\prime 1} \cdots E\left(P_{m}\right)=$ $D_{2}^{\prime m-1}$.

The configuration graph of the exceptional divisor of $\pi: U \longrightarrow \mathbb{C}^{2}$ is a linear graph with $m$ vertices. We will represent this graph on a vertical line. We represent the divisors $E\left(P_{1}\right), \cdots, E\left(P_{m}\right)$ from top to bottom. We connect the vertices by a segment since the divisors intersect. We add an edge at the top and at the bottom with arrows since they represent $E_{1}$ and $E_{2}$ but not exceptional divisors. To keep the information on the subdivision, we decorate the vertex corresponding to $E\left(P_{i}\right)$ with $q_{i}$ on the edge above the vertex and $p_{i}$ on the edge under the vertex. Usually we don't write the decorations for $E_{1}$ and $E_{2}$, but they appear in the computations as $(0,1)$ and $(1,0)$. The number $d$ 


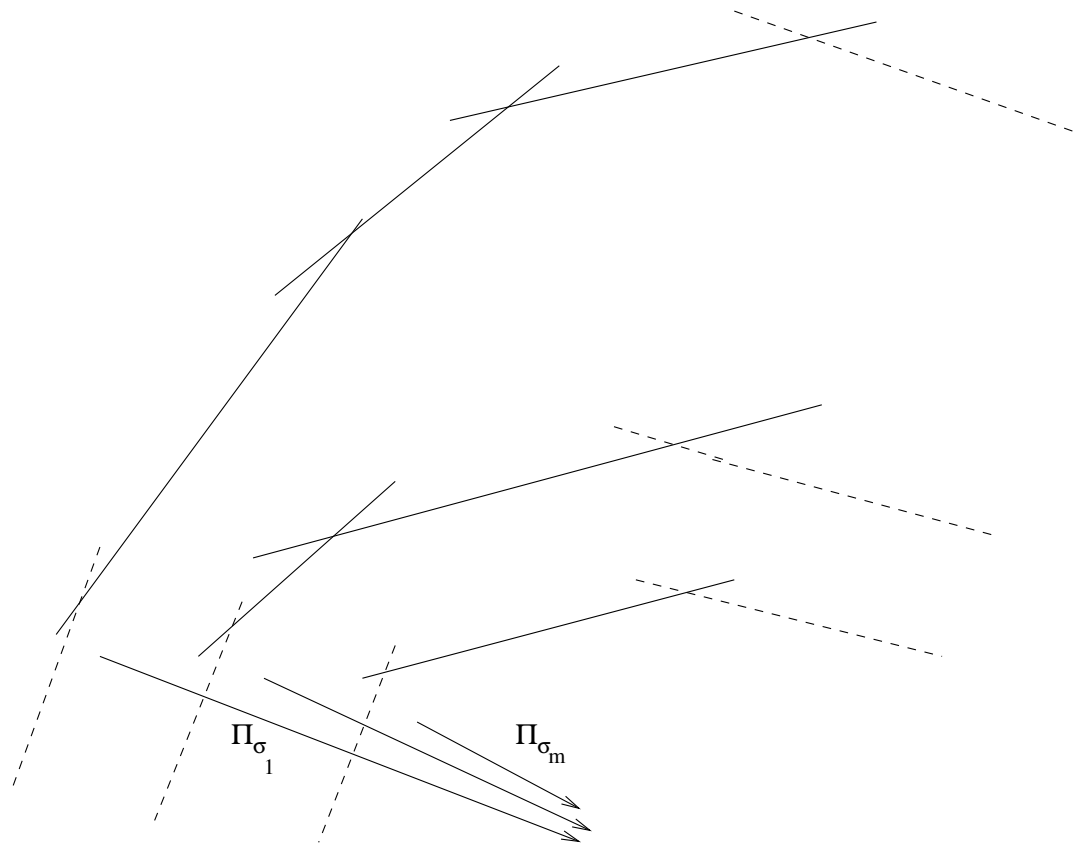

FiguRE 7

attached to a cone is computed as the edge determinant of the corresponding edge, ie. the product of the numbers on the edge minus the product of the numbers adjacent to the edge.
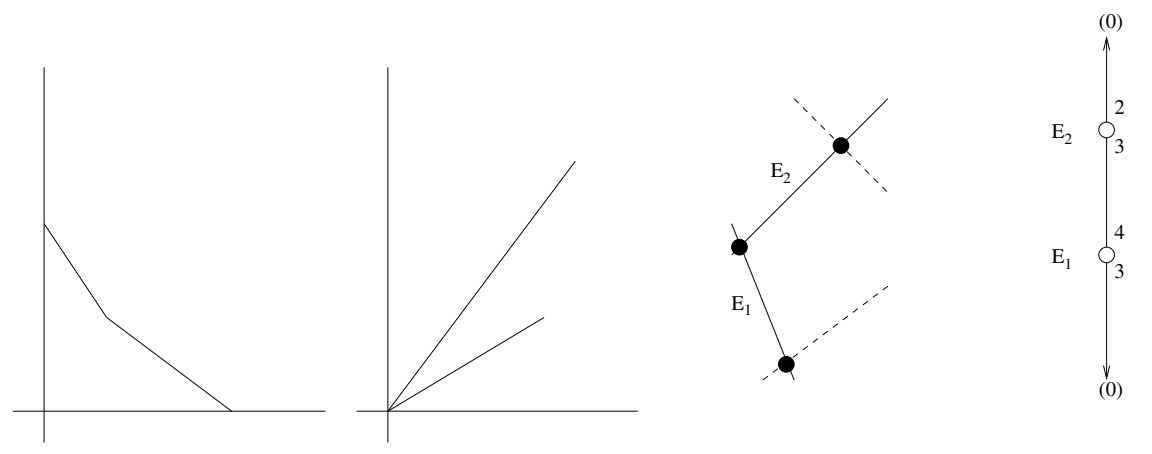

\section{FiguRE 8}

Each edge of the dual graph corresponds to a quotient singularity, of type the determinant of the edge. For the top and bottom edge it is $q_{1}$ and $p_{m}$ respectively.

EXAMPlE 3.3. We start with a Newton polygon with two faces with equations $3 \alpha+2 \beta=12$ and $3 \alpha+4 \beta=21$. We obtain the set of 
primitive vectors $\left\{P_{1}={ }^{t}(3,4), P_{2}={ }^{t}(3,2)\right\}$. We set as before $P_{0}=$ $(0,1)$ and $P_{3}=(1,0)$. We consider the cones $\sigma_{2}=$ cone $\left(P_{0}, P_{2}\right)$ and $\sigma_{2}^{\prime}=\operatorname{cone}\left(P_{2}, P_{3}\right)$. We have a toric variety $U_{2}$ which is the gluing of $U_{\sigma_{2}}$ and $U_{\sigma_{2}^{\prime}}$ and a birational map $\pi_{2}$ from $U_{2}$ to $\mathbb{C}^{2}$ with one exceptional divisor $E_{2}$ and $\pi_{2} \mid E_{2}$ is the restriction of the morphism

$$
\begin{array}{ccc}
\mathbb{C}^{2} & \longrightarrow & U_{\sigma_{2}} \\
\left(t_{1}, t_{2}\right) & & \mapsto
\end{array} \quad\left(x=t_{2}^{3}, y=t_{1} t_{2}^{2}\right)
$$

Now we split the cone $\sigma_{2}$ in $\sigma_{1}=\operatorname{cone}\left(P_{0}, P_{1}\right)$ and $\sigma_{1}^{\prime}=\operatorname{cone}\left(P_{1}, P_{2}\right)$. We have a toric variety $U$ which is the gluing of $U_{\sigma_{1}}, U_{\sigma_{1}^{\prime}}$ and $U_{\sigma_{2}^{\prime}}$ and a birational map $\pi$ from $U$ to $\mathbb{C}^{2}$ with two exceptional divisors $E_{1}$ and $E_{2}$ intersecting each other. The restriction of $\pi$ to $E_{2}$ is the restriction of $\pi_{2}$ to $E_{2}$. The restriction of $\pi$ to $E_{1}$ is the restriction to $E_{1}$ of the morphism

$$
\begin{gathered}
\mathbb{C}^{2} \\
\left(t_{1}, t_{2}\right)
\end{gathered} \quad \begin{gathered}
U_{\sigma_{1}} \\
\mapsto
\end{gathered} \quad \begin{gathered}
\longrightarrow \mathbb{C}^{2} \\
\left.\mapsto=t_{2}^{3}, y=t_{1} t_{2}^{4}\right)
\end{gathered}
$$

The toric variety $U$ is singular with 3 quotient singularities.

We represent the two exceptional divisors by two vertices with an edge connecting them since they intersect. We represent the lines $D_{2}^{1}$ on $U_{\sigma_{1}}$ and $D_{3}^{\prime 2}$ on $U_{\sigma_{2}^{\prime}}$ by an arrow decorated with $(0)$. They are not exceptional divisors.

3.2. Resolution of germs of plane curves. Let us begin by an example:

EXAMPLE 3.4. Consider the germ

$$
f(x, y)=x^{3}-y^{2}
$$

The Newton polygon of $f$ has one face with equation $2 \alpha+3 \beta=$ 6. We consider the two cones $\sigma=\operatorname{cone}\left({ }^{t}(0,1),{ }^{t}(2,3)\right)$ and $\sigma^{\prime}=$ cone $\left({ }^{t}(2,3),{ }^{t}(1,0)\right)$. The gluing of $U_{\sigma}$ and $U_{\sigma^{\prime}}$ gives a toric variety $U$ and a birational morphism from $U$ to $\mathbb{C}^{2}$. There is one exceptional divisor $E_{1}$ and the restriction of $\pi$ on $E_{1}$ is given by the restriction of the morphism

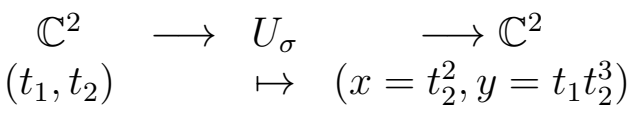

We have

$$
f\left(t_{2}^{2}, t_{1} t_{2}^{3}\right)=t_{2}^{6}\left(1-t_{1}^{2}\right)
$$

which means

$$
\pi^{*} f=\tilde{C}+6 E\left(P_{1}\right)
$$


where $\tilde{C}$ is the proper transform which intersects transversally the divisor $E_{1}$ in one point and is smooth.

The Newton tree associated to this germ is

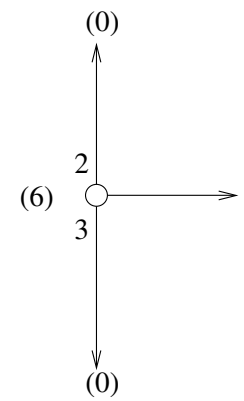

FiguRE 9

The vertex represents the divisor $E_{1}$. It is decorated with (6) which is the multiplicity of $f$ on this divisor. The numbers 2 and 3 represent the quotient singularities in $U_{\sigma}$ and $U_{\sigma^{\prime}}$, and the arrow represents the strict transform of $C$.

Let $f$ be a given complex analytic function of two variables defined on an open neighborhood of the origin such that $f(0,0)=0$.

We can consider the Newton polygon of $f$. Let $S \in \mathcal{N}(f)$. Assume the line supporting $S$ has equation $p \alpha+q \beta=N$, then we associate to $S$ the vector ${ }^{t}(p, q)$. Then to $\mathcal{N}(f)$, we associate $\left\{P_{1}, \cdots, P_{m}\right\}$. It gives us a simplicial cone subdivision $\Sigma$ of $N^{+}$. Then we can associate a toric variety $U$ and a birational morphism $\pi: U \rightarrow \mathbb{C}^{2}$ such that $\pi^{-1}(0)=\cup_{i=1}^{i=m} E\left(P_{i}\right)$. Notice that if we forget about the decorations of the arrows and of the vertices, the graph associated to the Newton polygon of $f$ is the graph dual to the divisor $\pi^{-1}(0)$ with two arrows decorated with (0) at the top and the bottom.

Let in $f_{S}=c x^{a_{S}} y^{b_{S}} \prod_{l=1}^{k_{S}}\left(x^{q}-\mu_{l} y^{p}\right)^{\nu_{l}}$. The exceptional divisor intersects the proper transform $\tilde{C}$ at $k_{S}$ points. Let $\tilde{C}_{l}$ be the union of the components of $\tilde{C}$ which pass through $\left(\mu_{l}, 0\right)$. The divisor $\pi^{*} f$ is given by

$$
\pi^{*} f=\sum_{i=1}^{m} \sum_{l=1}^{k_{i}} \tilde{C}_{i, l}+\sum_{i=0}^{m-1} N_{i} E\left(P_{i}\right) .
$$

If the germ is non degenerate, the components of the proper transform are smooth and transversal to the $E\left(P_{i}\right)$. And the vertices of Newton tree of $f$ represent the $E\left(P_{i}\right)$ and the arrows not decorated with (0) represent the components of $\tilde{C}$. 
If the germ is degenerate: Now if $\tilde{C}_{l}$ is not smooth or doesn't intersect transversally with $E(P)$, we consider $\left(\mu_{l}, 0\right)$ as the origin in $\mathbb{C}^{2}$ identifying $E(P)$ with $E_{1}$. If $\Pi_{\left(p, q, \mu_{l}\right)}^{*}(f)$ is not in good coordinates, we perform a change of variables which leaves $E(P)$ fixed. We consider the Newton polygon of $f_{\Pi_{\left(p, q, \mu_{l}\right)}}$ and the corresponding morphism $\pi_{1}: U_{1} \rightarrow U$. And we do the process again until we get the strict transform of $C$, smooth and transverse to the exceptionnal divisor.

Now recall the following (cf. [13],[26]).

Definition 3.5. A toroidal variety is a pair $(X, B)$ where $X$ is an algebraic variety and $B$ a Zariski closed subset such that for any $x \in X$ there exist a toric variety $\left(V_{x}, D_{x}\right)$ with a fixed 0-dimensional orbit $x^{\prime}$ and neighbourhoods $U_{x}, U_{x^{\prime}}$ in complex analytic topology of $x$ and $x^{\prime}$ respectively in $X$ and $V_{x}$ such that $\left(U_{x}, U_{x} \cap B\right)=\left(U_{x^{\prime}}, D_{x} \cap U_{x^{\prime}}\right)$.

With his we have the following:

Theorem 3.6. There exists a toroidal variety $\bar{U}$ and a birational morphism $\bar{\pi}: \bar{U} \rightarrow\left(\mathbb{C}^{2}, 0\right)$ such that

$$
\bar{\pi}^{*} f=\sum \tilde{C}_{n}+\sum N_{m} E_{m}
$$

such that the strict transforms of $f$ are smooth, this divisor has normal crossing and the Newton tree of $f$ is the dual graph of this divisor and the $N_{m}$ are the decorations of the corresponding vertices. The singularities of $\bar{U}$ are on the intersections of the divisors $E_{m}$, they are quotient singularities given by the edge determinant of the corresponding edge in the Newton tree. In particular $\bar{U}$ (and the Newton tree) determine the dual graph of smooth resolution via standard resolution of cyclic quotient singularities of $\bar{U}$

Proof. Let $\bar{U}$ be the variety obtained after the final step iteration of toric blow ups corresponding to subdivisions of the first quadrant corresponding to the Newton diagrams and changes to good systems of coordinates at the points intersection of the proper preimage of the zero set of the power series which singularitiy get resolved with the exceptional set of the toric blow up. Let $\cup E_{m}$ be the union of the proper preimages of the exceptional sets of all iterations. For each step, the toric blow up preserves toric structure, but good change of coordinates may destroy toric structure only at the smooth point of an exceptional curve at which this coordinate change is made. Denote them $P$ and $E_{P}$ respectively. Toric blow up at $P$, produces toric variety with respect to the toric structure in the new coordinate system. The proper preimage of $E_{P}$ intersects the exceptional set of the toric blow up at $P$ at smooth point. The boundary divisor consists of two smooth at 
this point, curves (i.e. the proper preimage of $E_{P}$ and the exceptional curve) and hence has obvious toric structure.

REMARK 3.7. The variety $\bar{U}$ together with the uniformization of sets $U_{\sigma}$ providing the cover of $\bar{U}$ also has the canonical structure of orbifold (cf. [1]) or stack. The resulting orbifold is not a global quotient in general. This provides the alternative category in which one has canonical resolution of singularities of $f$

REMARK 3.8. In the next section we shall consider the problem of extendability of 2-forms on the abelian covers $z_{i}^{m_{i}}=f_{i}$ where $f_{i}$ are irreducible components of a germ $f=0$ (cf. 4.1). After pull back this abelian cover on $\bar{U}$ the problem becomes about holomorphic extention of forms on resolution of singularities of the abelian cover of $\bar{U}$ ramified over the exceptional set of $\bar{U}$. Since singularities of $\bar{U}$ are cyclic quotients with exceptional curves near such singularity forming at most two orbits in resolution, one adds to exceptional curves of $\bar{U}$ only curves intersected by at most two other components of exceptional set i.e. resolution of quotient singularities does not add "rupture" curves. Such components in smooth resolution do not add restrictions on extendability (cf. [5]) Hence extendability of forms on abelian covers enough to check only on the exceptional curves which appear on $\bar{U}$.

We shall finish this section with calculation in terms of the Newton tree of the multiplicities of the pull back on the canonical resolution $\bar{U}$ of holomorphic on $\mathbb{C}^{2} 2$-form. If $v$ is a vertex on the Newton tree, denote by $\nu_{v}=$ mult $_{E} \pi^{*}(d x \wedge d y)$, if $E$ is the divisor corresponding to $v$.

Proposition 3.9. (1) If the vertex $v$ corresponds to a face of the Newton polygon of $f$ and is decorated by $(q, p)$ then $\nu_{v}=p+q$.

(2) If the vertex $v$ with nearby decorations $(q, p)$ has preceding vertex $v_{0}$ with nearby decorations $\left(q_{0}, p_{0}\right)$, then $\nu_{v}=\nu_{v_{0}} p+\delta$, where $\delta=q-p p_{0} q_{0}$.

Proof. We can compute the differential using the Newton map.

Consider the Newton map:

$$
x=x_{1}^{p} \mu_{k}^{p^{\prime}}, y=x_{1}^{q}\left(y_{1}+\mu_{k}^{q^{\prime}}\right)
$$

Then

$$
d x=p x_{1}^{p-1} \mu_{k}^{p^{\prime}} d x_{1}, d y=q x_{1}^{q-1}\left(y_{1}+\mu_{k}^{q^{\prime}}\right) d x_{1}+x_{1}^{q} d y_{1}
$$

Then

$$
d x \wedge d y=p x_{22}^{p+q-1} \mu_{k}^{p^{\prime}} d x_{1} \wedge d y_{1}
$$


which proves the first part of the proposition and

$$
x^{\nu_{v}-1} d x \wedge d y=p x_{1}^{\nu_{v} p+\delta-1} \mu_{k}^{p^{\prime}} d x_{1} \wedge d y_{1}
$$

because the Newton map is in this case

$$
x=x_{1}^{p} \mu_{k}^{p^{\prime}}, y=x_{1}^{q}\left(y_{1}+\mu_{k}^{q^{\prime}}\right)
$$

\section{Polytopes of QuAsi-Adjunction}

In this section, we will show how to compute explicitly polytopes of quasi-adjunction using Newton trees.

We first recall some definitions and results concerning polytopes of quasi-adjunction [5].

4.1. Ideals and polytopes of quasi-adjunction. Let $B$ be a small ball about the origin in $\mathbb{C}^{2}$ and let $\mathcal{C}$ be a germ of a plane curve having at 0 a singularity with $r$ branches . Let $f_{1}(x, y) \cdots f_{r}(x, y)=0$ be a local equation of this curve (each $f_{i}$ is assumed to be irreducible). An abelian cover of type $\left(m_{1}, \cdots, m_{r}\right)$ of $\partial B$ is the link of complete intersection surface singularity

$$
V_{m_{1}, \cdots, m_{r}}: z_{1}^{m_{1}}=f_{1}(x, y), \cdots, z_{r}^{m_{r}}=f_{r}(x, y)
$$

The covering map is given by $p:\left(z_{1}, \cdots, z_{r}, x, y\right) \rightarrow(x, y)$.

An ideal of quasi-adjunction of type $\left(j_{1}, \cdots, j_{r} \mid m_{1}, \cdots, m_{r}\right)$ is the ideal in the local ring of the singularity of $\mathcal{C}$ consisting of germs $\phi$ such that the 2-form:

$$
\omega_{\phi}=\frac{\phi z_{1}^{j_{1}} \cdots z_{r}^{j_{r}} d x \wedge d y}{z_{1}^{m_{1}-1} \cdots z_{r}^{m_{r}-1}}
$$

extends to a holomorphic form on a resolution of the singularity of $V_{m_{1}, \cdots, m_{r}}$.

Let

$$
\mathcal{U}=\left\{\left(x_{1}, \cdots, x_{r}\right) \in \mathbb{R}^{r}, 0 \leq x_{i}<1\right\}
$$

be the unit cube with coordinates corresponding to $f_{1}, \cdots, f_{r}$. Consider an ideal of quasi-adjunction $\mathcal{A}$. There is a unique polytope $\mathcal{P}(\mathcal{A})$ opensubset in $\mathcal{U}$ such that: For $\left(m_{1}, \cdots, m_{r}\right) \in \mathbb{Z}^{r}$ and $\left(j_{1}, \cdots, j_{r}\right) \in \mathbb{Z}^{r}$ with $0 \leq j_{i}<m_{i}, 1 \leq i \leq r$

$$
\mathcal{A} \subseteq \mathcal{A}\left(j_{1}, \ldots, j_{r} \mid m_{1}, \ldots, m_{r}\right) \Leftrightarrow\left(\frac{j_{1}+1}{m_{1}}, \cdots \frac{j_{r}+1}{m_{r}}\right) \in \mathcal{P}(\mathcal{A})
$$

A face of quasi-adjunction is a face of the boundary of the polytope $\mathcal{P}(\mathcal{A})$. It follows that it can be characterized as follows. Let $E_{i}$ be the exceptional curves of an embedded resolution $\pi: \tilde{\mathbb{C}}^{2} \rightarrow \mathbb{C}^{2}$ of $f_{1} \cdot \ldots \cdot f_{r}=$ 
0. Let $N_{i, k}=\operatorname{mult}_{E_{k}} \pi^{*}\left(f_{i}\right)$ be the multiplicity of pullback of $f_{i}$ to $\tilde{\mathbb{C}}^{2}$, $\nu_{k}=$ mult $_{E_{k}} \pi^{*}(d x \wedge d y)$ and for a germ $\phi \in \mathcal{O}_{O}, e_{k}(\phi)=$ mult $_{E_{k}} \pi^{*}(\phi)$. Then the face of quasi-adjunction containing $\wp=\left(\frac{j_{1}+1}{m_{1}}, \ldots, \frac{j_{r}+1}{m_{r}}\right) \in \mathcal{U}$ is the face $\alpha$ of the boundary of the set of points satisfying:

$$
\sum_{i} N_{i, k} x_{i}>\sum_{i} N_{i, k}-e_{k}(\phi)-\nu_{k}-1
$$

for all $\phi$ in the ideal of quasi-adjunction $\mathcal{A}\left(j_{1}, . ., j_{r} \mid m_{1}, \ldots, m_{r}\right)$ (and such that $\wp \in \alpha)$. In particular for $\left(j_{1}^{\prime}, \ldots, j_{r}^{\prime} \mid m_{1}^{\prime}, \ldots, m_{r}^{\prime}\right)$ for which the corresponding point satisfies (3) the form $\omega_{\phi}$ extends over all $E_{i}$. However for $\left(j_{1}^{\prime}, \ldots, j_{r}^{\prime} \mid m_{1}^{\prime}, \ldots, m_{r}^{\prime}\right)$ on the face itself there exist $\phi$ in the ideal of quasi-adjunction for which $\omega_{\phi}$ has pole on one of the exceptional curves.

4.2. Computation of faces of quasi-adjunction. Let $f_{1}, \cdots, f_{r}$ a collection of irreducible germs. We consider the Newton tree associated to $f_{1} f_{2} \cdots f_{r}$. Denote by $\mathcal{V}$ the set of vertices of the Newton tree. To each $v \in \mathcal{V}$ we associate the linear form

$$
l_{v}(s)=\sum_{i} N_{v, i}\left(s_{i}-1\right)+\nu_{v}
$$

where $N_{v, i}$ is the intersection multiplicity of $f_{i}$ with a curvette $C_{v}$ and $\nu_{v}$ is computed by Proposition 3.9.

Theorem 4.1. (1) Let $\phi$ be a germ, then the polytope of quasiadjunction associated to $\phi$ is defined by the set of inequalities

$$
l_{v}(s)>-e_{v}(\phi)
$$

for all $v \in \mathcal{V}$, where $e_{v}(\phi)$ is the intersection multiplicity of $\phi$ with a curvette $C_{v}$.

(2) For all $v \in \mathcal{V}$ there exist $\phi$ and a face of the polytope of quasiadjunction associated to $\phi$ with equation

$$
l_{v}(s)=-e_{v}(\phi)
$$

The first part of the theorem is a consequence of the discussion of part 3 which says that there is a bijection between the vertices of the Newton tree and the rupture divisors in a resolution, and Proposition 3.1 of [5], which says that we only need those divisors to compute the polytopes of quasi-adjunction.

The proof of the second part will occupy the remain of the section. 
4.2.1. The case $r=1$. As we have seen in [5], the polytopes of quasiadjunction $c$ (which in this case are called the constant of quasi-adjunction) are such that $1-c$ is an exponent between 0 and 1 . We will recover Saito's theorem. We will compute exponents instead of constants of quasi-adjunction.

Let $f$ be an irreducible germ. Its Newton tree is as Figure 10 .

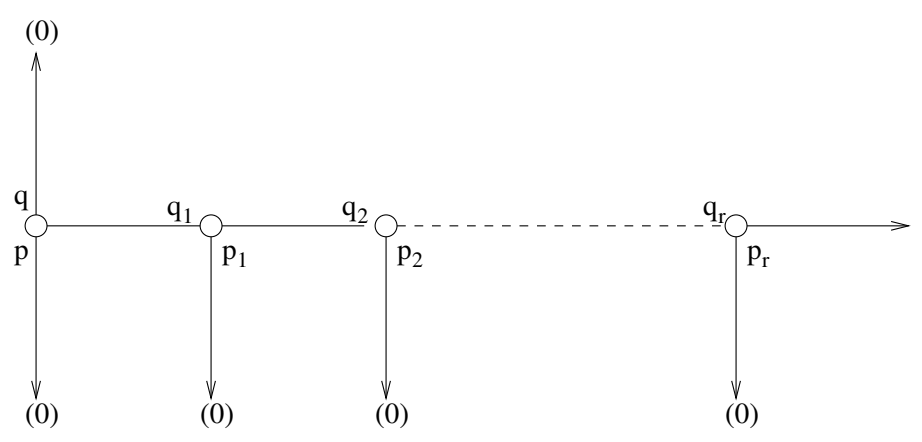

Figure 10

We denote $(q, p)=\left(q_{0}, p_{0}\right)$, the decorations of the first vertex. We assume $q_{0}>p_{0}$. We denote $\delta_{i}=q_{i}-p_{i} p_{i-1} q_{i-1}$.

We have indexed the vertices from 0 to $r$. The first part of theorem 4.1 says that the constant of quasi-adjunction associated to $\phi$ is given by $1-\epsilon(\phi)$ where

$$
\epsilon(\phi)=\min _{i=0, \cdots, r} \frac{\nu_{i}+e_{i}(\phi)}{p_{i} q_{i} \prod_{i+1}^{r} p_{j}} .
$$

We denote by

$$
\epsilon_{i}(\phi)=\frac{\nu_{i}+e_{i}(\phi)}{p_{i} q_{i} \prod_{i+1}^{r} p_{j}}
$$

and we call it the invariant of the vertex $v_{i}$ associated to $\phi$.

We will define a set of $\phi$ which gives all the exponents and such that the invariant along the horizontal path is decreasing to the minimum and then increasing. We need some technical lemma.

Lemma 4.2. For $i$ from 0 to $r$, we have

$$
p_{i} q_{i}-\nu_{i}>0
$$

Proof. For $i=0$, we have $p_{0} q_{0}-\nu_{0}=p_{0} q_{0}-p_{0}-q_{0}>0$ since $p_{0} \geq 2$.

Assume that $p_{i} q_{i}-\nu_{i}>0$.

$$
\begin{gathered}
p_{i+1} q_{i+1}-\nu_{i+1}=p_{i+1}\left(q_{i} p_{i} p_{i+1}+\delta_{i+1}\right)-\left(\nu_{i} p_{i+1}+\delta_{i+1}\right) \\
p_{i+1} q_{i+1}-\nu_{i+1}=p_{i+1}\left(p_{i} q_{i} p_{i+1}-\nu_{i}\right)+\left(p_{i+1}-1\right) \delta_{i+1}>p_{i} q_{i}-\nu_{i}>0 \\
25
\end{gathered}
$$


Definition 4.3. We consider irreducible germs $C_{0}^{\prime}, C_{0}, C_{1}, \cdots, C_{r}$ with Newton trees as in Figure 11.

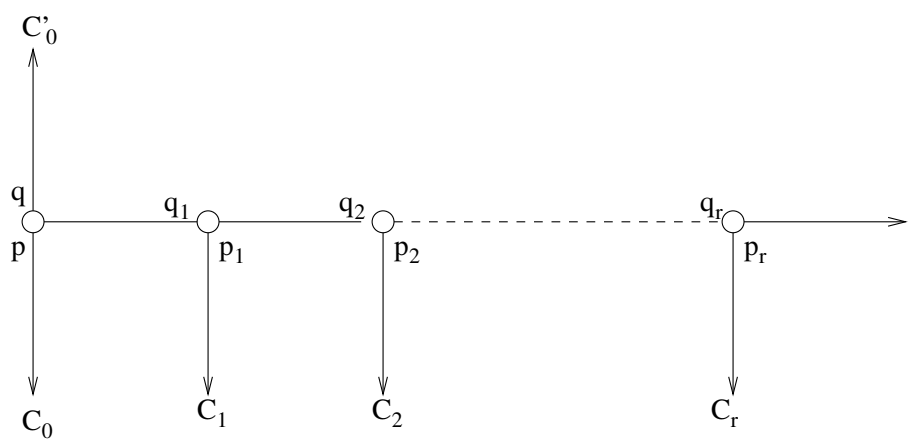

FIGURE 11

We define

$$
\phi=C_{0}^{\prime a} C_{0}^{b} C_{1}^{c_{1}} \cdots C_{r}^{c_{r}}
$$

We assume

$$
\forall i, 1 \leq i \leq r, 0 \leq c_{i}<p_{i}
$$

Define

$$
\begin{gathered}
A_{0}=a p_{0}+b q_{0} \\
A_{i+1}=A_{i} p_{i+1}+c_{i+1} q_{i+1}, i=0, \cdots r-1
\end{gathered}
$$

Lemma 4.4. For $i$ from 0 to $r-1$, we have

$$
\epsilon_{i}(\phi)-\epsilon_{i+1}(\phi)=\frac{\delta_{i+1}}{q_{i} q_{i+1} \prod_{i}^{r} p_{j}}\left(\nu_{i}-p_{i} q_{i}+A_{i}\right)
$$

Proof.

$$
\begin{gathered}
\nu_{i} q_{i+1}-\nu_{i+1} p_{i} q_{i}=\nu_{i}\left(p_{i} q_{i} p_{i+1}+\delta_{i+1}\right)-\left(\nu_{i} p_{i+1}+\delta_{i+1}\right) p_{i} q_{i} \\
\nu_{i} q_{i+1}-\nu_{i+1} p_{i} q_{i}=\delta_{i+1}\left(\nu_{i}-p_{i} q_{i}\right)
\end{gathered}
$$

We have to compute $q_{i+1} e_{i}(\phi)-p_{i} q_{i} e_{i+1}(\phi)$.

$$
\begin{gathered}
e_{i}(\phi)=\left(a p_{0}+b q_{0}\right) \prod_{1}^{i} p_{j}+q_{1} c_{1} \prod_{2}^{i} p_{j}+\cdots+q_{i} c_{i}+p_{i} q_{i}\left(c_{i+1}+\cdots+c_{r} \prod_{i+1}^{r-1} p_{j}\right) \\
e_{i+1}(\phi)=\left(a p_{0}+b q_{0}\right) \prod_{1}^{i+1} p_{j}+q_{1} c_{1} \prod_{26}^{i+1} p_{j}+\cdots+q_{i} c_{i} p_{i+1}+q_{i+1} c_{i+1}
\end{gathered}
$$




$$
+p_{i+1} q_{i+1}\left(c_{i+2}+\cdots+c_{r} \prod_{i+2}^{r-1} p_{j}\right)
$$

Then

$$
q_{i+1} e_{i}(\phi)-p_{i} q_{i} e_{i+1}(\phi)=\delta_{i+1} A_{i}
$$

Lemma 4.5. If $A_{i_{0}}<p_{i_{0}} q_{i_{0}}-\nu_{i_{0}}$, then $\forall i, i_{0} \leq i \leq r, A_{i}<p_{i} q_{i}-\nu_{i}$.

Proof. Assume $A_{i}<p_{i} q_{i}-\nu_{i}$.

$$
\begin{aligned}
A_{i+1}= & A_{i} p_{i+1}+c_{i+1} q_{i+1}<\left(p_{i} q_{i}-\nu_{i}\right) p_{i+1}+q_{i+1}\left(p_{i+1}-1\right)= \\
& q_{i+1}-\nu_{i+1}+p_{i+1} q_{i+1}-q_{i+1}=p_{i+1} q_{i+1}-\nu_{i+1}
\end{aligned}
$$

Next proposition will be used in all the remain of the article.

Proposition 4.6. Let $\phi$ be as in definition 4.3. Then

$$
\epsilon(\phi)=\epsilon_{i_{0}}(\phi)
$$

where $i_{0}$ is such that for $0 \leq i<i_{0}, A_{i}>p_{i} q_{i}-\nu_{i}$ and $A_{i_{0}}<p_{i_{0}} q_{i_{0}}-\nu_{i_{0}}$. Moreover, the sequence $\epsilon_{i}(\phi)$ is decreasing for $i \leq i_{0}$ and increasing for $i \geq i_{0}$.

Proof. If, for $0 \leq i \leq i_{0}-1$, we have $A_{i}>p_{i} q_{i}-\nu_{i}$, then

$$
\epsilon_{i}(\phi)>\epsilon_{i+1}(\phi)
$$

Then for $0 \leq i \leq i_{0}-1$

$$
\epsilon_{i}(\phi)>\epsilon_{i_{0}}(\phi)
$$

We have for $i_{0} \leq i \leq r, A_{i}<p_{i} q_{i}-\nu_{i}$ and

$$
\epsilon_{i}(\phi)<\epsilon_{i+1}(\phi)
$$

M. Saito [23] has proven the following

Theorem 4.7. Let $f$ be an irreducible plane singularity with Newton tree as in Figure 10

Then the exponents between 0 and 1 are given by the following formula

$$
e_{i}\left(k_{1}, k_{2}, k_{3}\right)=\frac{1}{\prod_{i+1}^{r} p_{j}}\left(\frac{k_{1}}{p_{i}}+\frac{k_{2}}{q_{i}}\right)+\frac{k_{3}}{\prod_{i+1}^{r} p_{j}}
$$

where $0<k_{1}<p_{i}$, $0<k_{2}<q_{i}, \frac{k_{1}}{p_{i}}+\frac{k_{2}}{q_{i}}<1,0 \leq k_{3}<\prod_{i+1}^{r} p_{j}$ for $0 \leq i \leq r$.

We prove that 
Proposition 4.8. For each $i$ and $k_{1}, k_{2}, k_{3}$, there exists $\phi$ as in proposition 4.6 such that $e_{i}\left(k_{1}, k_{2}, k_{3}\right)=\epsilon_{i}(\phi)=\epsilon(\phi)$.

Proof. We have to prove that for each $i, 0 \leq i \leq r$ and for each $\left(k_{1}, k_{2}, k_{3}\right)$ there exists $\phi$ such that

$$
\frac{1}{\prod_{i+1}^{r} p_{j}}\left(\frac{k_{1}}{p_{i}}+\frac{k_{2}}{q_{i}}\right)+\frac{k_{3}}{\prod_{i+1}^{r} p_{j}}=\epsilon_{i}(\phi)
$$

and

$$
\epsilon_{i}(\phi)=\epsilon(\phi)
$$

We can write

$$
\begin{gathered}
\nu_{i}=q_{i}-p_{i}\left(p_{i-1} q_{i-1}-\nu_{i-1}\right) \\
e_{i}(\phi)=\alpha_{i} p_{i}+\beta_{i} q_{i}+\gamma_{i} p_{i} q_{i}
\end{gathered}
$$

Then

$$
\frac{\nu_{i}+e_{i}(\phi)}{p_{i} q_{i} \prod_{i+1}^{r} p_{j}}=\frac{1}{\prod_{i+1}^{r} p_{j}}\left(\frac{\beta_{i}+1}{p_{i}}+\frac{\alpha_{i}-p_{i-1} q_{i-1}+\nu_{i-1}}{q_{i}}+\gamma_{i}\right)
$$

Let

$$
\alpha_{i}=p_{i-1} q_{i-1}-\nu_{i-1}+k_{2}, \beta_{i}=k_{1}-1, \gamma_{i}=k_{3} .
$$

We have

$$
\alpha_{i} p_{i}+\beta_{i} q_{i}=k_{2} p_{i}+k_{1} q_{i}-\nu_{i}<p_{i} q_{i}-\nu_{i}
$$

Now we show that we can find $\left(a, b, c_{1}, \cdots, c_{r}\right)$ with $0 \leq c_{i}<p_{i}$ for $1 \leq i \leq r$, such that

$$
\phi=C_{0}^{\prime a} C_{0}^{b} C_{1}^{c_{1}} \cdots C_{r}^{c_{r}} .
$$

We have $0 \leq k_{3}<\prod_{i+1}^{r} p_{j}$, then we can write

$$
\gamma_{i}=c_{i+1}+c_{i+2} p_{i+1}+\cdots+c_{r} p_{i+1} \cdots p_{r-1}
$$

with $0 \leq c_{l}<p_{l}$ for $i+1 \leq l \leq r$.

We will show that there exists $\left(a, b, c_{1}, \cdots, c_{i}\right)$ positive integers such that $\alpha_{i}=A_{i}$ and we set $\beta_{i}=c_{i}<p_{i}$. We need the following

Lemma 4.9. Let $p, q$ positive integers prime to each other. Let a be a positive integer $a=p q+k$ with $-p-q<k$. Then there exist $0 \leq n, 0 \leq m<p$ such that $a=n p+m q$.

Proof. Choose a pair $(n, m) \in \mathbb{Z}^{2}$ such that $a=n p+m q$. One can choose $m$ such that $0 \leq m<p$. If $a=p q+k$ with $k>-p-q$ then $n \geq 0$.

Lemma 4.10. For $0 \leq i \leq r$, we have $\nu_{i} \leq p_{i}+q_{i}$. 
Proof. For $i=0$, we have $\nu_{0}=p_{0}+q_{0}$. For $0<i$, $\nu_{i}=\nu_{i-1} p_{i}+\delta_{i-1}=\nu_{i-1} p_{i}+q_{i}-p_{i-1} q_{i-1} p_{i}=\left(\nu_{i-1}-p_{i-1} q_{i-1}\right) p_{i}+q_{i} \leq p_{i}+q_{i}$

We have

$$
\alpha_{i}=p_{i-1} q_{i-1}-\nu_{i-1}+k_{2} .
$$

with $k_{2} \geq 0$. Then the equation $\alpha_{i}=\alpha_{i, 1} p_{i-1}+\beta_{i, 1} q_{i-1}$ admits a solution in positive integers with $\beta_{i-1}<p_{i-1}$.

We have

$$
\alpha_{i, 1}>p_{i-2} q_{i-2}-\nu_{i-2}
$$

Since

$$
\begin{gathered}
\alpha_{i, 1} p_{i-1}+\beta_{i, 1} q_{i-1}>p_{i-1} q_{i-1}-\nu_{i-1} \\
\alpha_{i, 1} p_{i-1}>\left(p_{i-1}-\beta_{i, 1}\right) q_{i-1}-\nu_{i-1} \\
\alpha_{i, 1} p_{i-1}>\left(p_{i-1}-\beta_{i, 1}\right)\left(p_{i-2} q_{i-2} p_{i-1}+\delta_{i-1}\right)-\left(\nu_{i-2} p_{i-1}+\delta_{i-1}\right) \\
\alpha_{i, 1} p_{i-1}>\left(p_{i-1}-\beta_{i, 1}-1\right) \delta_{i-1}+\left(p_{i-2} q_{i-2}-\nu_{i-2}\right) p_{i-1}
\end{gathered}
$$

Then we can write

$$
\alpha_{i, 1}=\alpha_{i, 2} p_{i-2}+\beta_{i, 2} q_{i-2}
$$

with $0 \leq \beta_{i, 2}<p_{i-2}$ and $\alpha_{i, 2}>p_{i-3} q_{i-3}-\nu_{i-3}$.

We define $\alpha_{i}=A_{i-1}, \beta_{i}=c_{i}, \alpha_{i, 1}=A_{i-2}, \beta_{i, 1}=c_{i-1} \cdots \alpha_{i, i-1}=$ $A_{0}, \beta_{i, i-1}=c_{1}$. We have $A_{0}>p_{0} q_{0}-\nu_{0}$ and we write $A_{0}=a p_{0}+b q_{0}$.

REMARK 4.11. (1) The last proposition prove that the numbers $e_{i}\left(k_{1}, k_{2}, k_{3}\right)$ are exponents between 0 and 1 . Then the bounds for $k_{1}, k_{2}, k_{3}$ imply that they are all the exponents between 0 and 1 and then we have a new proof of Saito theorem.

(2) We have

$$
\min _{i} \frac{\nu_{i}}{p_{i} q_{i} \prod_{i+1} p_{j}}=\frac{\nu_{0}}{p_{0} q_{0} \prod_{1} p_{j}}=\epsilon_{0}(1)=\epsilon(1) .
$$

This is the minimum of the exponents and is called the logcanonical threshold.

(3) For all $i \neq 01-\frac{\nu_{i}}{p_{i} q_{i} \prod_{i+1} p_{j}}=1-\epsilon_{i}(1)$ is an exponent. In fact we can write

$p_{i} q_{i} \prod_{i+1} p_{j}-q_{i}+p_{i}\left(p_{i-1} q_{i-1}-\nu_{i-1}\right)=q_{i}\left(p_{i}-1\right)+p_{i}\left(p_{i-1} q_{i-1}-\nu_{i-1}\right)+p_{i} q_{i}\left(\prod_{i+1} p_{j}-1\right)$

This is not true that for any $\phi$ the minimum $\frac{\nu_{i}+e_{i}(\phi)}{p_{i} q_{i} \prod_{i+1} p_{j}}$ is an exponent and $1-\frac{\nu_{l}+e_{l}(\phi)}{p_{l} q_{l} \prod_{l+1} p_{j}}$ is an exponent for $l \neq i$. One example is given by $\left(p_{0}, q_{0}\right)=(2,3),\left(p_{1}, q_{1}\right)=(2,13),\left(p_{2}, q_{2}\right)=$ 
$(2,53)$ and $\phi=C_{1} C_{2}$. We have $23 / 24<50 / 52<102 / 106$, $23 / 24$ is an exponent, but $2 / 52$ and $4 / 106$ are not. This remark is connected to the monodromy conjecture and the recent work of Nemethi and Veys [20].

We can show that for each vertex there is an exponent obtained by this vertex and no other.

Proposition 4.12. For each $i$, there exist $\phi$ such that $\epsilon_{i}(\phi)=\epsilon(\phi)$ and for all $\phi^{\prime}$ and $j \neq i, \epsilon(\phi) \neq \epsilon_{j}\left(\phi^{\prime}\right)$.

To prove this result we need some lemma.

Lemma 4.13. For all $j>i$, and all $k<i$, we have

$$
\frac{1}{\prod_{i+1} p_{l}}\left(\frac{1}{p_{i}}+\frac{1}{q_{i}}\right) \neq \frac{1}{\prod_{j+1} p_{l}}\left(\frac{k_{1}}{p_{j}}+\frac{k_{2}}{q_{j}}+k_{3}\right)
$$

and

$$
\frac{1}{\prod_{i+1} p_{l}}\left(\frac{1}{p_{i}}+\frac{1}{q_{i}}\right) \neq \frac{1}{\prod_{k+1} p_{l}}\left(\frac{k_{1}}{p_{k}}+\frac{k_{2}}{q_{k}}+k_{3}\right)
$$

Proof. Assume that there exists $\left(k_{1}, k_{2}, k_{3}\right)$ such that

$$
\frac{1}{\prod_{i+1} p_{l}}\left(\frac{1}{p_{i}}+\frac{1}{q_{i}}\right)=\frac{1}{\prod_{j+1} p_{l}}\left(\frac{k_{1}}{p_{j}}+\frac{k_{2}}{q_{j}}+k_{3}\right)
$$

that is

$$
\frac{1}{\prod_{i+1}^{j} p_{l}}\left(\frac{1}{p_{i}}+\frac{1}{q_{i}}\right)=\left(\frac{k_{1}}{p_{j}}+\frac{k_{2}}{q_{j}}+k_{3}\right)
$$

We have $\frac{k_{1}}{p_{j}}+\frac{k_{2}}{q_{j}}+k_{3} \geq \frac{1}{p_{j}}$ and $\frac{1}{\prod_{i+1}^{j} p_{l}}\left(\frac{1}{p_{i}}+\frac{1}{q_{i}}\right)<\frac{1}{p_{j}}$. Then the first assertion is proved.

Assume that there exists $\left(k_{1}, k_{2}, k_{3}\right)$ such that

$$
\frac{1}{\prod_{i+1} p_{l}}\left(\frac{1}{p_{i}}+\frac{1}{q_{i}}\right)=\frac{1}{\prod_{k+1} p_{l}}\left(\frac{k_{1}}{p_{k}}+\frac{k_{2}}{q_{k}}+k_{3}\right),
$$

that is

$$
\frac{1}{p_{i}}+\frac{1}{q_{i}}=\frac{1}{\prod_{k+1}^{i} p_{l}}\left(\frac{k_{1}}{p_{k}}+\frac{k_{2}}{q_{k}}+k_{3}\right) .
$$

On the left hand side, the denominator is $p_{i} q_{i}$. On the right hand side, the denominator is a divisor of $q_{k} \prod_{k}^{i} p_{l}$. But $p_{i} q_{i}>q_{k} \prod_{k}^{i} p_{l}$. The second assertion is proved.

Now we can prove proposition 
Proof. Consider a vertex of the Newton tree $v$ decorated with $\left(p_{i}, q_{i}\right)$.

Consider the exponent $\frac{1}{\prod_{i+1} p_{l}}\left(\frac{1}{p_{i}}+\frac{1}{q_{i}}\right)$. We found a $\phi$ such that

$$
\frac{1}{\prod_{i+1} p_{l}}\left(\frac{1}{p_{i}}+\frac{1}{q_{i}}\right)=\frac{\nu_{i}+e_{i}(\phi)}{p_{i} q_{i} \prod_{i+1} p_{l}}<\frac{\nu_{j}+e_{j}(\phi)}{p_{j} q_{j} \prod_{j+1} p_{l}}
$$

for $j \neq i$. Assume there exits $m \neq i$ and $\phi^{\prime}$ such that

$$
\frac{1}{\prod_{i+1} p_{l}}\left(\frac{1}{p_{i}}+\frac{1}{q_{i}}\right)=\frac{\nu_{m}+e_{m}\left(\phi^{\prime}\right)}{p_{m} q_{m} \prod_{m+1} p_{l}}<\frac{\nu_{j}+e_{j}\left(\phi^{\prime}\right)}{p_{j} q_{j} \prod_{j+1} p_{l}}
$$

for $j \neq m$. Since $\frac{\nu_{m}+e_{m}\left(\phi^{\prime}\right)}{p_{m} q_{m} \prod_{m+1} p_{l}}$ is $1-c$ with $c$ a constant of quasiadjunction, it is an exponent and so can be written $\frac{1}{\prod_{j+1} p_{l}}\left(\frac{k_{1}}{p_{j}}+\frac{k_{2}}{q_{j}}+k_{3}\right)$. This is not possible using the previous lemma. Then the exponent $\frac{1}{\prod_{i+1} p_{l}}\left(\frac{1}{p_{i}}+\frac{1}{q_{i}}\right)$ is given by the vertex $v$ and no other.

4.2.2. Induction step. We want to prove the theorem by induction. We need the following proposition.

Proposition 4.14. The intersection of the polytopes of quasi-adjunction of $f_{1} \cdots f_{r}$ with the hyperplane $s_{1}=1$ are the polytopes of quasiadjunction of $f_{2} \cdots f_{r}$.

Proof. The polytopes of quasi-adjunction of $f_{1} \cdots f_{r}$ are given by inequalities

$$
\sum_{i}^{r} N_{v, i}\left(s_{i}-1\right)+\nu_{v}+e_{v}(\phi)>0
$$

where $v$ runs over all the vertices of the Newton tree of $f_{1} \cdots f_{r}$. We have to show that when $s_{1}=1$ we only need inequalities with $v$ running over the vertices of the Newton tree of $f_{2} \cdots f_{r}$.

To delete $f_{1}$ we cut an horizontal edge $e$ arising from a vertex $v$. We have two cases. Either the edge $e$ ends by an arrow and we do not delete any vertices except eventually $v$, or we delete a line of vertices. We study this case.

Let $v$ and $v^{\prime}$ be the two vertices ending $e$. If we cut $e$ the vertex $v^{\prime}$ is deleted. We prove that the inequality

$$
\sum_{2}^{r} N_{v, i}\left(s_{i}-1\right)+\nu_{v}+e_{v}(\phi)>0
$$

implies

$$
\sum_{2}^{r} N_{v^{\prime}, i}\left(s_{i}-1\right)+\nu_{v^{\prime}}+e_{v^{\prime}}(\phi)>0
$$




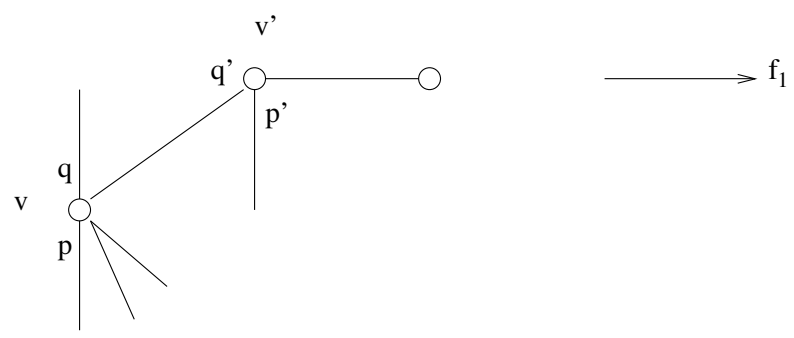

FiguRe 12

The vertex $v$ is decorated with $(q, p)$ (neither one on $e$ ) and the vertex $v^{\prime}$ is decorated with $\left(q^{\prime}, p^{\prime}\right)$ (with $q^{\prime}$ on $e$ ), we denote by $\delta=q^{\prime}-p q p^{\prime}>0$. We have, for $i \neq 1, N_{v^{\prime}, i}=p^{\prime} N_{v, i}, \nu_{v^{\prime}}=\nu_{v} p^{\prime}+\delta$, and we can write

$$
\begin{gathered}
e_{v}(\phi)=a+\beta b p q+c p q, e_{v^{\prime}}(\phi)=a p^{\prime}+\alpha b p^{\prime}+c q^{\prime} \\
\sum_{2}^{r} N_{v^{\prime}, i}\left(s_{i}-1\right)+\nu_{v^{\prime}}+e_{v^{\prime}}(\phi) \\
=\left(\sum_{2}^{r} N_{v, i}\left(s_{i}-1\right)+\nu_{v}+e_{v}(\phi)\right) p^{\prime}+p^{\prime} b(\alpha-\beta p q)+(c+1) \delta \\
>\sum_{2}^{r} N_{v, i}\left(s_{i}-1\right)+\nu_{v}+e_{v}(\phi)
\end{gathered}
$$

for all $\phi$. The same computation shows that if $v^{\prime \prime}$ is the vertex attached to $v^{\prime}$ on the Newton tree of $f_{1}$, we have the inequality

$$
\sum_{2}^{r} N_{v^{\prime}, i}\left(s_{i}-1\right)+\nu_{v^{\prime}}+e_{v^{\prime}}(\phi)>0
$$

implies

$$
\sum_{2}^{r} N_{v^{\prime \prime}, i}\left(s_{i}-1\right)+\nu_{v^{\prime \prime}}+e_{v^{\prime \prime}}(\phi)>0
$$

Now if $v$ is not a vertex on the Newton tree of $f_{2} \cdots f_{r}$ the inequality corresponding to $v$ is not needed from Proposition 3.1 in [5].

Now we can prove the theorem. We use induction on the number $r$ of irreducible branches. It is true for $r=1$. Assume it is true for any set of $r-1$ of irreducible functions. Consider $f_{1}, \cdots f_{r}$. We consider the intersection of the polytopes of quasi-adjunction of $f_{1} \cdots f_{r}$ with the hyperplane $s_{1}=1$. This gives the polytopes of quasi-adjunction of $f_{2} \cdots f_{r}$. Then all the vertices on the Newton tree of $f_{2} \cdots f_{r}$ contribute. 
If we have all the vertices of $f_{1} \cdots f_{r}$ we are done. Otherwise we consider all the possible choices of $r-1$ functions amongst $f_{1}, \cdots, f_{r}$. All the vertices of the Newton tree of $f_{1} \cdots f_{r}$ will appear except in the two cases:

(1) $r=2$ and $v$ is decorated with $(q, 1)$

(2) $r=3$ and $v$ is decorated with $(1,1)$.

4.2.3. Exceptional cases. The rest of the section is devoted to the proof of these particular cases.

We have to consider those cases separately.

\section{Case I.1}

This is the case where $v$ is connected by an edge to the arrows representing $f_{1}$ and $f_{2}$.

We consider first the case where we have only one vertex.
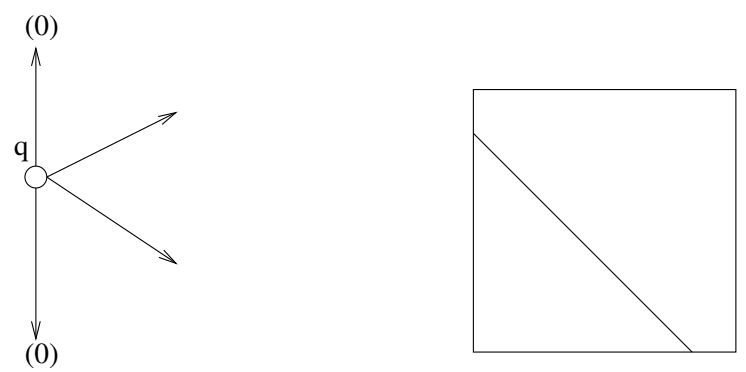

FIGURE 13

The log canonical threshold is $q\left(s_{1}-1\right)+q\left(s_{2}-1\right)+q+1>0$.

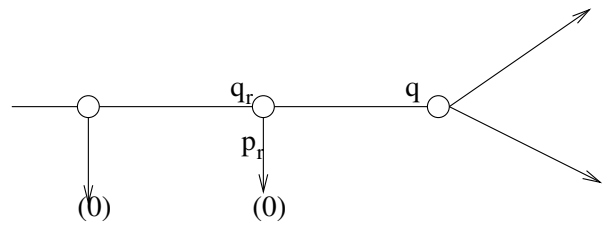

FiguRE 14

The polytopes of quasi-adjunction are given by the following inequalities:

$$
\begin{array}{ccccc}
q_{0} p_{0} \prod_{1}^{r} p_{j}\left(s_{1}-1\right) & +q_{0} p_{0} \prod_{1}^{r} p_{j}\left(s_{2}-1\right) & +\nu_{0} & +e_{0}(\phi) & >0 \\
\ldots & & & & \\
q_{r} p_{r}\left(s_{1}-1\right) & +q_{r} p_{r}\left(s_{2}-1\right) & +\nu_{r} & +e_{r}(\phi) & >0 \\
q\left(s_{1}-1\right) & +q\left(s_{2}-1\right) & +\nu & +e(\phi) & >0
\end{array}
$$

All the hyperplanes defined by the equality are parallel. 
If we could erase the last inequality, we would have the inequalities defining the polytopes of quasi-adjunction in the case the two germs separate on the vertex decorated with $\left(q_{r}, p_{r}\right)$ (Figure 15).

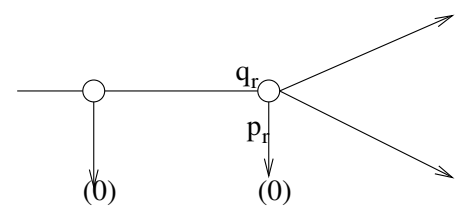

FIGURE 15

But we know that the number of intersections of the polytopes of quasi-adjunction with the line $s_{1}=s_{2}$ inside the cube is the Milnor number. The Milnor numbers of the two singularities differ by $q-p_{r} q_{r}$. Then we need all the inequalities.

EXAMPLE 4.15. (see Figure 16)

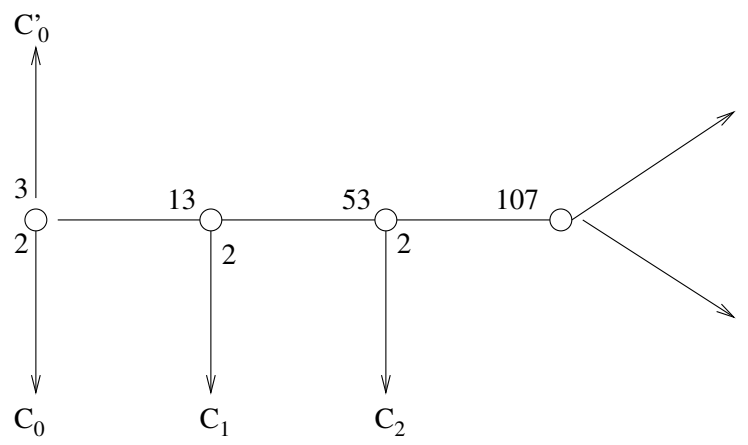

Figure 16

The polytopes of quasi-adjunction are given by

$$
\begin{array}{cc}
24\left(s_{1}-1\right)+24\left(s_{2}-1\right)+5+e_{0}(\phi) & >0 \\
52\left(s_{1}-1\right)+52\left(s_{2}-1\right)+11+e_{1}(\phi) & >0 \\
106\left(s_{1}-1\right)+106\left(s_{2}-1\right)+23+e_{2}(\phi) & >0 \\
107\left(s_{1}-1\right)+107\left(s_{2}-1\right)+24+e(\phi) & >0
\end{array}
$$

We take $\phi=C_{0}^{\prime} C_{1} C_{2}$ and the corresponding face of quasi-adjunction is given by

$$
107\left(s_{1}-1\right)+107\left(s_{2}-1\right)+111=0
$$

\section{Case I.2}

We first consider the following case (see Figure 17).

We consider $\phi=1$. 


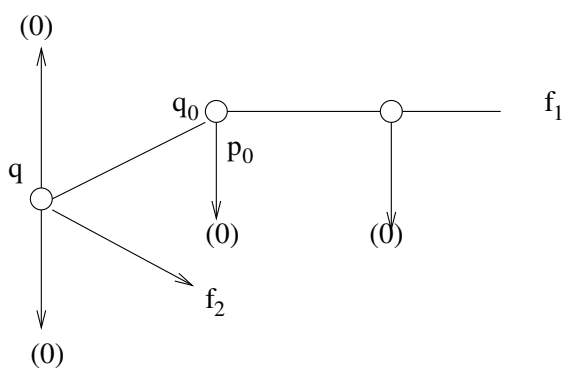

FiguRe 17

We have the set of following inequalities to compute the log-canonical wall.

$$
\begin{array}{ccc}
q \prod_{0}^{r} p_{j}\left(s_{1}-1\right) & +q\left(s_{2}-1\right) & +\nu>0 \\
q_{0} p_{0} \prod_{1}^{r} p_{j}\left(s_{1}-1\right) & +q p_{0}\left(s_{2}-1\right) & +\nu_{0}>0 \\
q_{i} p_{i} \prod_{i+1}^{r} p_{j}\left(s_{1}-1\right) & +q \prod_{0}^{i} p_{j}\left(s_{2}-1\right) & +\nu_{i}>0
\end{array}
$$

Since we have for all $i$ from 0 to $r-1$,

$$
1-\frac{\nu_{i}}{p_{i} q_{i} \prod_{i+1} p_{j}}>1-\frac{\nu_{i+1}}{p_{i+1} q_{i+1} \prod_{i+2} p_{j}}
$$

and

$$
1-\frac{\nu_{i}}{q \prod_{0}^{i} p_{j}}>1-\frac{\nu_{i+1}}{q \prod_{0}^{i+1} p_{j}}
$$

the inequality $q_{0} p_{0} \prod_{1}^{r} p_{j}\left(s_{1}-1\right)+q p_{0}\left(s_{2}-1\right)+\nu_{0}>0$ implies the inequalities $q_{i} p_{i} \prod_{i+1}^{r} p_{j}\left(s_{1}-1\right)+q \prod_{0}^{i} p_{j}\left(s_{2}-1\right)+\nu_{i}>0$ for $i$ from 1 to $r$. We are left with the two inequalities

$$
\begin{array}{ccc}
q \prod_{0}^{r} p_{j}\left(s_{1}-1\right) & +q\left(s_{2}-1\right) & +\nu>0 \\
q_{0} p_{0} \prod_{1}^{r} p_{j}\left(s_{1}-1\right) & +q p_{0}\left(s_{2}-1\right) & +\nu_{0}>0
\end{array}
$$

We compute the intersection of the two lines $q \prod_{0}^{r} p_{j}\left(s_{1}-1\right)+q\left(s_{2}-\right.$ 1) $+\nu=0$ and $q_{0} p_{0} \prod_{1}^{r} p_{j}\left(s_{1}-1\right)+q p_{0}\left(s_{2}-1\right)+\nu_{0}=0$, which is $\left(s_{1}=1-\frac{1}{\prod_{0}^{r} p_{j}}, s_{2}=1-\frac{1}{q}\right)$, which means that the log-canonical wall is a breaking line and that the equation $q \prod_{0}^{r} p_{j}\left(s_{1}-1\right)+q\left(s_{2}-1\right)+\nu=0$ does occur.

Now we consider (see Figure 18)

We chose $\phi$ as in proposition 4.6 such that

$$
\max _{j}\left(1-\frac{\nu_{j}+e_{j}(\phi)}{p_{j} q_{j} \prod_{j+1} p_{l}}\right)=1-\frac{1}{\prod_{i+2} p_{l}}\left(\frac{1}{q_{i+1}}+\frac{1}{p_{i+1}}\right)
$$

That means that with the notation of Definition 4.3 we have $\phi=$ $C_{0}^{\prime a} C_{0}^{b} C_{1}^{c_{1}} \cdots C_{i}^{c_{i}}, e_{i}(\phi)=A_{i}=p_{i} q_{i}-\nu_{i}+1$. 


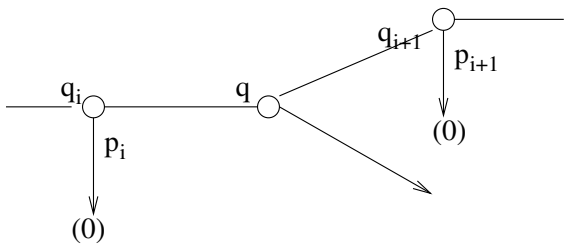

FiguRE 18

We have to consider

$$
\begin{gathered}
q_{j} p_{j} \prod_{j+1} p_{l}\left(s_{1}-1\right)+q_{j} p_{j} \prod_{j+1}^{i} p_{l}\left(s_{2}-1\right)+\nu_{j}+e_{j}(\phi)>0, j<i \\
q_{i} p_{i} \prod_{i+1} p_{l}\left(s_{1}-1\right)+q_{i} p_{i}\left(s_{2}-1\right)+\nu_{i}+e_{i}(\phi)>0 \\
q \prod_{i+1} p_{l}\left(s_{1}-1\right)+q\left(s_{2}-1\right)+\nu+e(\phi)>0 \\
q_{i+1} p_{i+1} \prod_{i+2} p_{l}\left(s_{1}-1\right)+q p_{i+1}\left(s_{2}-1\right)+\nu_{i+1}+e_{i+1}(\phi)>0 \\
q_{j} p_{j} \prod_{j+1} p_{l}\left(s_{1}-1\right)+q \prod_{i+1}^{j} p_{l}\left(s_{2}-1\right)+\nu_{j}+e_{j}(\phi)>0, j>i+1
\end{gathered}
$$

Since, for $j \geq i+1$

$$
1-\frac{\nu_{j}+e_{j}(\phi)}{p_{j} q_{j} \prod_{j+1} p_{l}}>1-\frac{\nu_{j+1}+e_{j+1}(\phi)}{p_{j+1} q_{j+1} \prod_{j+2} p_{l}}
$$

and

$$
1-\frac{\nu_{j}+e_{j}(\phi)}{q \prod_{i+1}^{j} p_{l}}>1-\frac{\nu_{j+1}+e_{j+1}(\phi)}{q \prod_{i+1}^{j+1} p_{l}},
$$

because $\nu_{j+1}+e_{j+1}(\phi)-\left(\nu_{j}+e_{j}(\phi)\right) p_{j+1}=\delta_{j+1}$. Then the inequality $q_{i+1} p_{i+1} \prod_{i+2} p_{l}\left(s_{1}-1\right)+q p_{i+1}\left(s_{2}-1\right)+\nu_{i+1}+e_{i+1}(\phi)>0$ implies the inequalities $q_{j} p_{j} \prod_{j+1} p_{l}\left(s_{1}-1\right)+q \prod_{i+1}^{j} p_{l}\left(s_{2}-1\right)+\nu_{j}+e_{j}(\phi)>0, j>$ $i+1$.

We have, for $j \leq i$,

$$
\begin{aligned}
& 1-\frac{\nu_{j}+e_{j}(\phi)}{p_{j} q_{j} \prod_{j+1}^{r} p_{l}}>1-\frac{\nu_{j-1}+e_{j-1}(\phi)}{p_{j-1} q_{j-1} \prod_{j}^{r} p_{l}} \\
& 1-\frac{\nu_{j}+e_{j}(\phi)}{p_{j} q_{j} \prod_{j+1}^{i} p_{l}}>1-\frac{\nu_{j-1}+e_{j-1}(\phi)}{p_{j-1} q_{j-1} \prod_{j}^{i} p_{l}}
\end{aligned}
$$

Then the inequality $q_{i} p_{i} \prod_{i+1} p_{l}\left(s_{1}-1\right)+q_{i} p_{i}\left(s_{2}-1\right)+\nu_{i}+e_{i}(\phi)>0$ implies the inequalities $q_{j} p_{j} \prod_{j+1} p_{l}\left(s_{1}-1\right)+q_{j} p_{j} \prod_{j+1}^{i} p_{l}\left(s_{2}-1\right)+\nu_{j}+$ $e_{j}(\phi)>0, j<i$.

Then we are left with the inequalities

$$
\begin{gathered}
q_{i} p_{i} \prod_{i+1} p_{l}\left(s_{1}-1\right)+q_{i} p_{i}\left(s_{2}-1\right)+\nu_{i}+e_{i}(\phi)>0 \\
q \prod_{i+1} p_{l}\left(s_{1}-1\right)+q\left(s_{2}-1\right)+\nu+e(\phi)>0 \\
q_{i+1} p_{i+1} \prod_{i+2} p_{l}\left(s_{1}-1\right)+q p_{i+1}\left(s_{2}-1\right)+\nu_{i+1}+e_{i+1}(\phi)>0 \\
36
\end{gathered}
$$


The second one implies the first one. In fact since $e_{i}(\phi)=p_{i} q_{i}-\nu_{i}+1=$ $e(\phi)$, we have

$$
\begin{gathered}
1-\frac{\nu_{i}+e_{i}(\phi)}{p_{i} q_{i} \prod_{i+1}^{r} p_{l}}<1-\frac{\nu+e(\phi)}{q \prod_{i+1}^{r} p_{l}} \\
1-\frac{\nu_{i}+e_{i}(\phi)}{p_{i} q_{i}}<1-\frac{\nu+e(\phi)}{q}
\end{gathered}
$$

Then finally we have to consider two inequalities

$$
\begin{gathered}
q \prod_{i+1} p_{l}\left(s_{1}-1\right)+q\left(s_{2}-1\right)+\nu+e(\phi)>0 \\
q_{i+1} p_{i+1} \prod_{i+2} p_{l}\left(s_{1}-1\right)+q p_{i+1}\left(s_{2}-1\right)+\nu_{i+1}+e_{i+1}(\phi)>0
\end{gathered}
$$

The intersection point of the lines $q \prod_{i+1} p_{l}\left(s_{1}-1\right)+q\left(s_{2}-1\right)+\nu+$ $e(\phi)=0$ and $q_{i+1} p_{i+1} \prod_{i+2} p_{l}\left(s_{1}-1\right)+q p_{i+1}\left(s_{2}-1\right)+\nu_{i+1}+e_{i+1}(\phi)=0$ is $\left(s_{1}=1-\frac{1}{\prod_{i+1}^{r} p_{j}}, s_{2}=1-\frac{1}{q}\right)$. Then we have a polytope of quasiadjunction with a broken line as face and the inequality $q \prod_{i+1} p_{l}\left(s_{1}-\right.$ 1) $+q\left(s_{2}-1\right)+\nu+e(\phi)=0$ does occur.

Example 4.16. (see Figure 19)

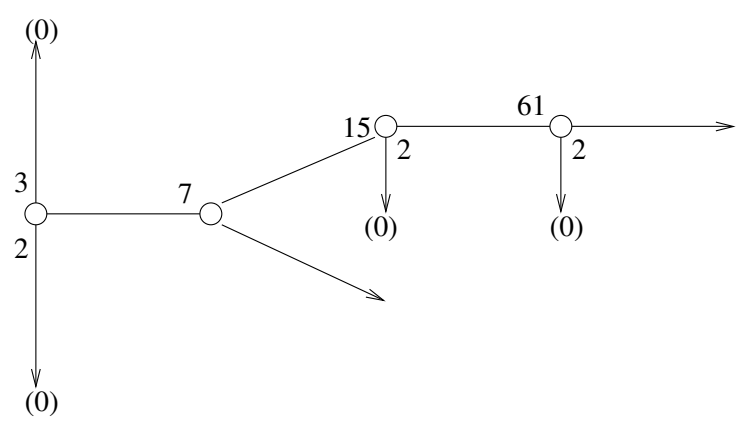

FIGURE 19

We have a face of quasi-adjunction given by

$$
28\left(s_{1}-1\right)+7\left(s_{2}-1\right)+8>0,60\left(s_{1}-1\right)+14\left(s_{2}-1\right)+17>0
$$

Case I.3 (See Figure 20)

The log-canonical wall is given by the equations

$$
\begin{array}{cccc}
q \prod_{0}^{r^{1}} p_{l}^{1}\left(s_{1}-1\right) & +q \prod_{0}^{r^{2}} p_{l}^{2}\left(s_{2}-1\right) & +\nu & >0 \\
p_{0}^{1} q_{0}^{1} \prod_{1}^{r_{1}^{1}} p_{l}^{1}\left(s_{1}-1\right) & +q p_{0}^{1} \prod_{0}^{r^{2}} p_{l}^{2}\left(s_{2}-1\right) & +\nu_{0}^{1} & >0 \\
p_{j}^{1} q_{j}^{1} \prod_{j+1}^{r_{1}^{1}} p_{l}^{1}\left(s_{1}-1\right) & +q \prod_{0}^{j} p_{l}^{1} \prod_{0}^{r^{2}} p_{l}^{2}\left(s_{2}-1\right) & +\nu_{j}^{1} & >0,0<j<r^{1} \\
q p_{0}^{2} \prod_{0}^{r_{1}^{1}} p_{l}^{1}\left(s_{1}-1\right) & +p_{0}^{2} q_{0}^{2} \prod_{1}^{r^{2}} p_{l}^{2}\left(s_{2}-1\right) & +\nu_{0}^{2} & >0 \\
q \prod_{0}^{k} p_{l}^{2} \prod_{0}^{r^{1}} p_{l}^{1}\left(s_{1}-1\right) & +p_{k}^{2} q_{k}^{2} \prod_{k+1}^{r^{2}} p_{l}^{2}\left(s_{2}-1\right) & +\nu_{k}^{2} & >0,0<k<r^{2} \\
& & &
\end{array}
$$




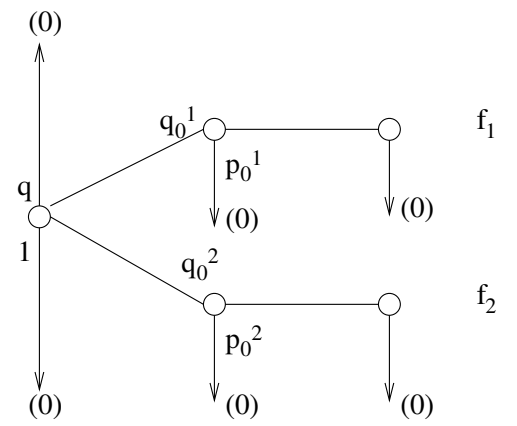

FigURE 20

We have

$$
1-\frac{\nu_{j}^{1}}{p_{j}^{1} q_{j}^{1} \prod_{j+1} p_{l}^{1}}>1-\frac{\nu_{j+1}^{1}}{p_{j+1}^{1} q_{j+1}^{1} \prod_{j+2} p_{l}^{1}}
$$

and

$$
1-\frac{\nu_{j}^{1}}{q \prod_{0}^{j} p_{l}^{1} \prod_{0}^{r^{2}} p_{l}^{2}}>1-\frac{\nu_{j+1}^{1}}{q \prod_{0}^{j+1} p_{l}^{1} \prod_{0}^{r^{2}} p_{l}^{2}}
$$

Then we have to consider the three inequalities

$$
\begin{array}{cccc}
q \prod_{0}^{r^{1}} p_{l}^{1}\left(s_{1}-1\right) & +q \prod_{0}^{r^{2}} p_{l}^{2}\left(s_{2}-1\right) & +\nu & >0 \\
p_{0}^{1} q_{0}^{1} \prod_{1}^{r^{1}} p_{l}^{1}\left(s_{1}-1\right) & +q p_{0}^{1} \prod_{0}^{r^{2}} p_{l}^{2}\left(s_{2}-1\right) & +\nu_{0}^{1} & >0 \\
q p_{0}^{2} \prod_{0}^{r^{1}} p_{l}^{1}\left(s_{1}-1\right) & +p_{0}^{2} q_{0}^{2} \prod_{1}^{r^{2}} p_{l}^{2}\left(s_{2}-1\right) & +\nu_{0}^{2} & >0
\end{array}
$$

We show that the three lines

(0) $q \prod_{0}^{r^{1}} p_{l}^{1}\left(s_{1}-1\right) \quad+q \prod_{0}^{r^{2}} p_{l}^{2}\left(s_{2}-1\right) \quad+\nu \quad=0$

(1) $p_{0}^{1} q_{0}^{1} \prod_{1}^{r^{1}} p_{l}^{1}\left(s_{1}-1\right)+q p_{0}^{1} \prod_{0}^{r^{2}} p_{l}^{2}\left(s_{2}-1\right) \quad+\nu_{0}^{1}=0$

(2) $q p_{0}^{2} \prod_{0}^{r^{1}} p_{l}^{1}\left(s_{1}-1\right) \quad+p_{0}^{2} q_{0}^{2} \prod_{1}^{r^{2}} p_{l}^{2}\left(s_{2}-1\right) \quad+\nu_{0}^{2}=0$

constitute the log-canonical wall.

We have

$$
1-\frac{\nu_{0}^{1}}{p_{0}^{1} q_{0}^{1} \prod_{1}^{r^{1}} p_{l}^{1}}>1-\frac{\nu}{q \prod_{0}^{r^{1}} p_{l}^{1}}
$$

Then the intersection of the line (1) with the line $\left\{s_{2}=1\right\}$ is greater than the intersection of the line (0) with $\left\{s_{2}=1\right\}$. The same for the intersection of (2) and $\left\{s_{1}=1\right\}$.

Now we show that the intersection of the lines (1) and (2) is on the same side of the line (0) than the origin. The intersection of (1) and 


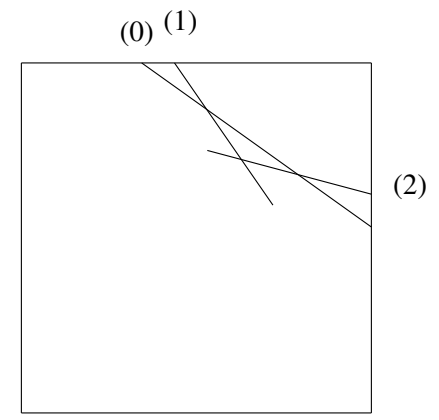

Figure 21

(2) is given by

$$
\begin{aligned}
& \left(q_{0}^{1} q_{0}^{2}-q^{2} p_{0}^{1} p_{0}^{2}\right) \prod_{0}^{r^{1}} p_{l}^{1}\left(s_{1}-1\right)+\nu_{0}^{1} q_{0}^{2}-\nu_{0}^{2} q p_{0}^{1}=0 \\
& \left(q_{0}^{1} q_{0}^{2}-q^{2} p_{0}^{1} p_{0}^{2}\right) \prod_{0}^{r^{2}} p_{l}^{2}\left(s_{2}-1\right)+\nu_{0}^{2} q_{0}^{1}-\nu_{0}^{1} q p_{0}^{2}=0
\end{aligned}
$$

We have

$$
\begin{gathered}
\left(q_{0}^{1} q_{0}^{2}-q^{2} p_{0}^{1} p_{0}^{2}\right)=q\left(\delta^{1} p_{0}^{2}+\delta^{2} p_{0}^{1}\right)+\delta^{1} \delta^{2} \\
\nu_{0}^{1} q_{0}^{2}-\nu_{0}^{2} q p_{0}^{1}=q\left(\delta^{1} p_{0}^{2}-\delta^{2} p_{0}^{1}\right)+\delta^{1} \delta^{2}+\nu p_{0}^{1} \delta^{2}
\end{gathered}
$$

A simple computation gives the result.

Then the log canonical wall is given by the three lines

$$
\begin{array}{ccccc}
(0) & q \prod_{0}^{r^{1}} p_{l}^{1}\left(s_{1}-1\right) & +q \prod_{0}^{r^{2}} p_{l}^{2}\left(s_{2}-1\right) & +\nu & =0 \\
(1) & p_{0}^{1} q_{0}^{1} \prod_{1}^{r^{1}} p_{l}^{1}\left(s_{1}-1\right) & +q p_{0}^{1} \prod_{0}^{r^{2}} p_{l}^{2}\left(s_{2}-1\right) & +\nu_{0}^{1}=0 \\
(2) & q p_{0}^{2} \prod_{0}^{r^{1}} p_{l}^{1}\left(s_{1}-1\right) & +p_{0}^{2} q_{0}^{2} \prod_{1}^{r^{2}} p_{l}^{2}\left(s_{2}-1\right) & +\nu_{0}^{2} & =0
\end{array}
$$

and the first vertex does occur.

ExAMPLE 4.17. (see Figure 22)

The log-canonical wall is given by the three inequalities

$$
\begin{aligned}
4\left(s_{1}-1\right)+4\left(s_{2}-1\right)+2 & >0 \\
10\left(s_{1}-1\right)+8\left(s_{2}-1\right)+5 & >0 \\
8\left(s_{1}-1\right)+10\left(s_{2}-1\right)+5 & >0
\end{aligned}
$$

Now we study the case where the separation occurs as in Figure 23.

We consider $\phi$ such that

$$
1-\frac{1}{\prod_{i+2}^{r^{1}} p_{l}^{1}}\left(\frac{1}{q_{i+1}^{1}}+\frac{1}{p_{i+1}^{1}}\right)=1-\frac{\nu_{i+1}^{1}+e_{i+1}^{1}(\phi)}{q_{i+1}^{1} p_{i+1}^{1} \prod_{i+2}^{r^{1}} p_{l}^{1}}
$$




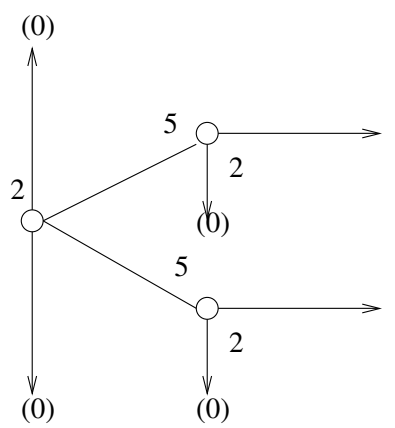

FiguRe 22
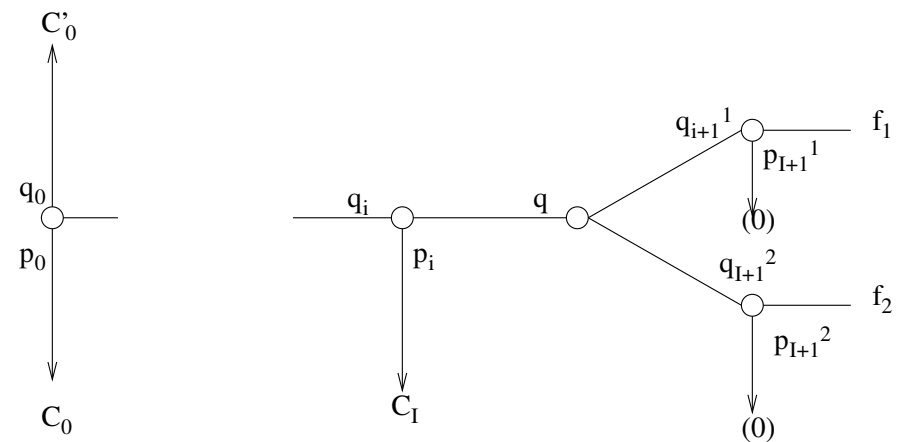

FiguRE 23

is $\max _{0 \leq j \leq r^{1}} 1-\frac{\nu_{j}^{1}+e_{j}^{1}(\phi)}{q_{j}^{1} p_{j}^{1} \prod_{j+1}^{r 1} p_{l}^{1}}$ and

$$
1-\frac{1}{\prod_{i+2}^{r^{2}} p_{l}^{2}}\left(\frac{1}{q_{i+1}^{2}}+\frac{1}{p_{i+2}^{2}}\right)=1-\frac{\nu_{i+1}^{2}+e_{i+1}^{2}(\phi)}{q_{i+1}^{2} p_{i+1}^{2} \prod_{i+2}^{r^{2}} p_{l}^{2}}
$$

is $\max _{0 \leq j \leq r^{2}} 1-\frac{\nu_{j}^{2}+e_{j}^{2}(\phi)}{q_{j}^{2} p_{j}^{2} \prod_{j+1}^{2} p_{l}^{2}}$.

This can be achieve with $\phi=C_{0}^{\prime a} C_{0}^{b} \cdots C_{i}^{c_{i}}$ with $A_{i}=p_{i} q_{i}-\nu_{i}+1$ with notations of 4.6 . 
We have to consider the inequalities

$$
\begin{gathered}
q_{j} p_{j} \prod_{j+1}^{r^{1}} p_{l}^{1}\left(s_{1}-1\right)+q_{j} p_{j} \prod_{j+1}^{r^{2}} p_{l}^{2}\left(s_{2}-1\right)+\nu_{j}+e_{j}(\phi)>0,0 \leq j<i \\
q_{i} p_{i} \prod_{i+1}^{r^{1}} p_{l}^{1}\left(s_{1}-1\right)+q_{i} p_{i} \prod_{i+1}^{r^{2}} p_{l}^{2}\left(s_{2}-1\right)+\nu_{i}+e_{i}(\phi)>0 \\
q \prod_{i+1}^{r^{1}} p_{l}^{1}\left(s_{1}-1\right)+q \prod_{i+1}^{r^{2}} p_{l}^{2}\left(s_{2}-1\right)+\nu+e(\phi)>0 \\
q_{i+1}^{1} p_{i+1}^{1} \prod_{i+2}^{r^{1}} p_{l}^{1}\left(s_{1}-1\right)+q p_{i+1}^{1} \prod_{i+1}^{r^{2}} p_{l}^{2}\left(s_{2}-1\right)+\nu_{i+1}^{1}+e_{i+1}^{1}(\phi)>0 \\
q p_{i+1}^{2} \prod_{i+1}^{r^{1}} p_{l}^{1}\left(s_{1}-1\right)+q_{i+1}^{2} p_{i+1}^{2} \prod_{i+2}^{r^{2}} p_{l}^{2}\left(s_{2}-1\right)+\nu_{i+1}^{2}+e_{i+1}^{2}(\phi)>0 \\
q_{n}^{1} p_{n}^{1} \prod_{n+1}^{r^{1}} p_{l}^{1}\left(s_{1}-1\right)+q \prod_{i+1}^{n} p_{l}^{1} \prod_{i+1}^{r^{2}} p_{l}^{2}\left(s_{2}-1\right)+\nu_{n}^{1}+e_{n}^{1}(\phi)>0, i+1<n \leq r^{1} \\
q \prod_{i+1}^{m} p_{l}^{2} \prod_{i+1}^{r^{1}} p_{l}^{1}\left(s_{1}-1\right)+q_{m} p_{m}^{2} \prod_{m+1}^{r^{2}} p_{l}^{2}\left(s_{2}-1\right)+\nu_{m}^{2}+e_{m}^{2}(\phi)>0, i+1<m \leq r^{2}
\end{gathered}
$$

where for $0 \leq j<i p_{j}=p_{j}^{1}=p_{j}^{2}$ and $q_{j}=q_{j}^{1}=q_{j}^{2}$.

As before, we don't need the two last sets of inequalities.

We have

and

$$
1-\frac{\nu_{j}+e_{j}(\phi)}{q_{j} p_{j} \prod_{j+1}^{r^{1}} p_{l}^{1}}>1-\frac{\nu_{j-1}+e_{j-1}(\phi)}{q_{j-1} p_{j-1} \prod_{j}^{r^{1}} p_{l}^{1}}
$$

$$
1-\frac{\nu_{j}+e_{j}(\phi)}{q_{j} p_{j} \prod_{j+1}^{r^{2}} p_{l}^{2}}>1-\frac{\nu_{j-1}+e_{j-1}(\phi)}{q_{j-1} p_{j-1} \prod_{j}^{r 2} p_{l}^{2}}
$$

Then we don't need either the first set of inequalities. We are left with

$$
\begin{gathered}
q_{i} p_{i} \prod_{i+1}^{r^{1}} p_{l}^{1}\left(s_{1}-1\right)+q_{i} p_{i} \prod_{r_{i+1}}^{r^{2}} p_{l}^{2}\left(s_{2}-1\right)+\nu_{i}+e_{i}(\phi)>0 \\
q \prod_{i+1}^{r^{1}} p_{l}^{1}\left(s_{1}-1\right)+q \prod_{i+1}^{r^{2}} p_{l}^{2}\left(s_{2}-1\right)+\nu+e(\phi)>0 \\
q_{i+1}^{1} p_{i+1}^{1} \prod_{i+2}^{r^{1}} p_{l}^{1}\left(s_{1}-1\right)+q p_{i+1}^{1} \prod_{i+1}^{r^{2}} p_{l}^{2}\left(s_{2}-1\right)+\nu_{i+1}^{1}+e_{i+1}^{1}(\phi)>0 \\
q p_{i+1}^{2} \prod_{i+1}^{r^{1}} p_{l}^{1}\left(s_{1}-1\right)+q_{i+1}^{2} p_{i+1}^{2} \prod_{i+2}^{r^{2}} p_{l}^{2}\left(s_{2}-1\right)+\nu_{i+1}^{2}+e_{i+1}^{2}(\phi)>0
\end{gathered}
$$

We show that we don't need the first inequality and that the three last give a polytope of quasi-adjunction. Then the second inequality does occur.

We have

$$
\begin{aligned}
& 1-\frac{\nu_{i}+e_{i}(\phi)}{q_{i} p_{i} \prod_{i+1}^{r^{1}} p_{l}^{1}}<1-\frac{\nu+e(\phi)}{q \prod_{i+1}^{r^{1}} p_{l}^{1}} \\
& 1-\frac{\nu_{i}+e_{i}(\phi)}{q_{i} p_{i} \prod_{i+1}^{r^{2}} p_{l}^{2}}<1-\frac{\nu+e(\phi)}{q \prod_{i+1}^{r^{2}} p_{l}^{2}}
\end{aligned}
$$

then the second inequality implies the first one.

We consider

$$
\begin{gathered}
(0) q \prod_{r_{1+1}}^{r^{1}} p_{l}^{1}\left(s_{1}-1\right)+q \prod_{i+1}^{r^{2}} p_{l}^{2}\left(s_{2}-1\right)+\nu+e(\phi)=0 \\
(1) q_{i+1}^{1} p_{i+1}^{1} \prod_{i+2}^{r^{1}} p_{l}^{1}\left(s_{1}-1\right)+q p_{i+1}^{1} \prod_{i+1}^{r^{2}} p_{l}^{2}\left(s_{2}-1\right)+\nu_{i+1}^{1}+e_{i+1}^{1}(\phi)=0 \\
(2) q p_{i+1}^{2} \prod_{i+1}^{r^{1}} p_{l}^{1}\left(s_{1}-1\right)+q_{i+1}^{2} p_{i+1}^{2} \prod_{41}^{r^{2}} p_{l}^{2}\left(s_{2}-1\right)+\nu_{i+1}^{2}+e_{i+1}^{2}(\phi)=0
\end{gathered}
$$


As before, we show that the abscissa of the intersection of line (1) with $\left\{s_{2}=1\right\}$ is greater than the abscissa of the intersection of line (0) with $\left\{s_{2}=1\right\}$. In fact, we have

$$
1-\frac{\nu_{i+1}+e_{i+1}(\phi)}{q_{i+1} p_{i+1} \prod_{i+2}^{r^{1}} p_{l}^{1}}>1-\frac{\nu+e(\phi)}{q \prod_{i+1}^{r^{1}} p_{l}^{1}}
$$

since

$$
(\nu+e(\phi)) q_{i+1}^{1}-q\left(\nu_{i+1}+e_{i+1}(\phi)\right)=\delta^{1}(e(\phi)+\nu-q)=\delta^{1}
$$

since $e(\phi)=p_{i} q_{i}-\nu_{i}+1$. Now, the intersection point of (1) and (2) is given by

$$
\begin{gathered}
\left(q_{i+1}^{1} q_{i+1}^{2}-q^{2} p_{i+1}^{1} p_{i+1}^{2}\right) \prod_{i+1}^{r^{1}} p_{l}^{1}\left(s_{1}-1\right)+ \\
\left.q_{i+1}^{2} \nu_{i+1}^{1}-q p_{i+1}^{1} \nu_{i+1}^{2}\right)+e_{i+1}^{1}(\phi) q_{i+1}^{2}-e_{i+1}^{2}(\phi) q p_{i+1}^{1}=0 \\
\left(q_{i+1}^{1} q_{i+1}^{2}-q^{2} p_{i+1}^{1} p_{i+1}^{2}\right) \prod_{i+1}^{r^{2}} p_{l}^{2}\left(s_{2}-1\right)+ \\
\left.q_{i+1}^{1} \nu_{i+1}^{2}-q p_{i+1}^{2} \nu_{i+1}^{1}\right)+e_{i+1}^{2}(\phi) q_{i+1}^{1}-e_{i+1}^{1}(\phi) q p_{i+1}^{2}=0
\end{gathered}
$$

We have

$$
\begin{gathered}
q_{i+1}^{1} q_{i+1}^{2}-q^{2} p_{i+1}^{1} p_{i+1}^{2}=q\left(\delta^{1} p_{i+1}^{2}+\delta^{2} p_{i+1}^{1}\right)+\delta^{1} \delta^{2} \\
\left.q_{i+1}^{2} \nu_{i+1}^{1}-q p_{i+1}^{1} \nu_{i+1}^{2}\right)=\delta^{1} p_{i+1}^{2}(\nu-q)+\delta^{2} q p_{i+1}^{1}+\delta^{1} \delta^{2} \\
e_{i+1}^{1}(\phi) q_{i+1}^{2}-e_{i+1}^{2}(\phi) q p_{i+1}^{1}=e(\phi) p_{i+1}^{1} \delta^{2}
\end{gathered}
$$

using the fact that $e(\phi)=p_{i} q_{i}-\nu_{i}+1$, we can show that the intersection point of the two lines (1) and (2) is on the same side of the line (0) than the origin.

EXAMPLE 4.18. (see Figure 24)

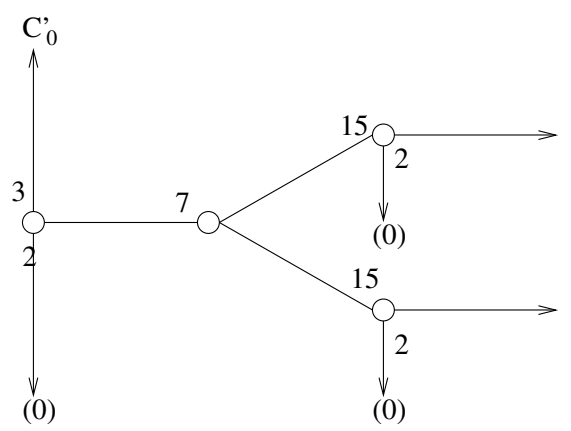

FiguRE 24 
The three inequalities give a polytope of quasi-adjunction.

$$
\begin{gathered}
14\left(s_{1}-1\right)+14\left(s_{2}-1\right)+8>0 \\
30\left(s_{1}-1\right)+28\left(s_{2}-1\right)+17>0 \\
28\left(s_{1}-1\right)+30\left(s_{2}-1\right)+17>0
\end{gathered}
$$

Then Case I is proved

\section{Case II}

We study the case where $r=3$ and the three germs are connected by a vertex decorated by $(1,1)$.

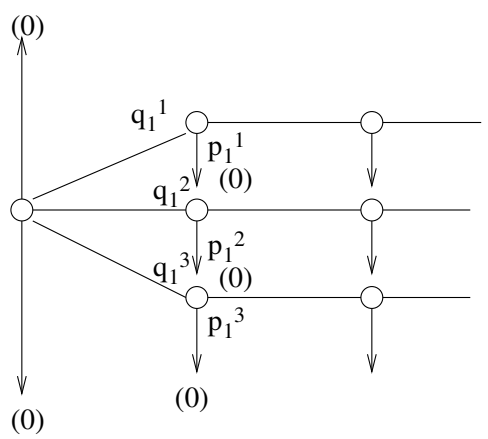

FigURE 25

We compute the log-canonical wall. We have to consider the following inequalities.

$$
\begin{gathered}
\prod_{1}^{r^{1}} p_{l}^{1}\left(s_{1}-1\right)+\prod_{1}^{r^{2}} p_{l}^{2}\left(s_{2}-1\right)+\prod_{1}^{r^{3}} p_{l}^{3}\left(s_{3}-1\right)+2>0 \\
q_{1}^{1} p_{1}^{1} \prod_{r^{1}}^{r^{1}} p_{l}^{1}\left(s_{1}-1\right)+p_{1}^{1} \prod_{1}^{r^{2}} p_{l}^{2}\left(s_{2}-1\right)+p_{1}^{1} \prod_{1}^{r^{3}} p_{l}^{3}\left(s_{3}-1\right)+\nu_{1}^{1}>0 \\
p_{1}^{2} \prod_{1}^{r^{1}} p_{l}^{1}\left(s_{1}-1\right)+q_{1}^{2} p_{1}^{2} \prod_{2}^{r^{2}} p_{l}^{2}\left(s_{2}-1\right)+p_{1}^{2} \prod_{1}^{r^{3}} p_{l}^{3}\left(s_{3}-1\right)+\nu_{1}^{2}>0 \\
p_{1}^{3} \prod_{1}^{r_{1}^{1}} p_{l}^{1}\left(s_{1}-1\right)+p_{1}^{3} \prod_{1}^{r^{2}} p_{l}^{2}\left(s_{2}-1\right)+q_{1}^{3} p_{1}^{3} \prod_{2}^{r^{3}} p_{l}^{3}\left(s_{3}-1\right)+\nu_{1}^{3}>0 \\
q_{i}^{1} p_{i}^{1} \prod_{i+1}^{r^{1}} p_{l}^{1}\left(s_{1}-1\right)+\prod_{1}^{i} p_{l}^{1} \prod_{1}^{r^{2}} p_{l}^{2}\left(s_{2}-1\right)+\prod_{1}^{i} p_{l}^{1} \prod_{1}^{r^{3}} p_{l}^{3}\left(s_{3}-1\right)+\nu_{i}^{1}>0,2 \leq i \leq r^{1} \\
\prod_{1}^{j} p_{l}^{2} \prod_{1}^{r_{1}^{1}} p_{l}^{1}\left(s_{1}-1\right)+q_{j}^{2} p_{j}^{2} \prod_{j+1}^{r^{2}} p_{l}^{2}\left(s_{2}-1\right)+\prod_{1}^{j} p_{l}^{2} \prod_{1}^{r^{3}} p_{l}^{3}\left(s_{3}-1\right)+\nu_{j}^{2}>0,2 \leq j \leq r^{2} \\
\prod_{1}^{k} p_{l}^{3} \prod_{1}^{r^{1}} p_{l}^{1}\left(s_{1}-1\right)+\prod_{1}^{k} p_{l}^{3} \prod_{1}^{r^{2}} p_{l}^{2}\left(s_{2}-1\right)+q_{k}^{3} p_{k}^{3} \prod_{k+1}^{r^{3}} p_{l}^{3}\left(s_{3}-1\right)+\nu_{k}^{3}>0,2 \leq k \leq r^{3}
\end{gathered}
$$

It is easy to check that the three last sets of inequality do not contribute to the log-canonical wall. We show that the four remaining inequalities all contribute to the log-canonical wall. Let

(0) $\prod_{1}^{r^{1}} p_{l}^{1}\left(s_{1}-1\right)+\prod_{1}^{r^{2}} p_{l}^{2}\left(s_{2}-1\right)+\prod_{1}^{r^{3}} p_{l}^{3}\left(s_{3}-1\right)+2=0$

(1) $q_{1}^{1} p_{1}^{1} \prod_{2}^{r^{1}} p_{l}^{1}\left(s_{1}-1\right)+p_{1}^{1} \prod_{1}^{r^{2}} p_{l}^{2}\left(s_{2}-1\right)+p_{1}^{1} \prod_{1_{3}}^{r^{3}} p_{l}^{3}\left(s_{3}-1\right)+\nu_{1}^{1}=0$

(2) $p_{1}^{2} \prod_{1}^{r_{1}^{1}} p_{l}^{1}\left(s_{1}-1\right)+q_{1}^{2} p_{1}^{2} \prod_{2}^{r^{2}} p_{l}^{2}\left(s_{2}-1\right)+p_{1}^{2} \prod_{1_{3}}^{r^{3}} p_{l}^{3}\left(s_{3}-1\right)+\nu_{1}^{2}=0$

(3) $p_{1}^{3} \prod_{1}^{r^{1}} p_{l}^{1}\left(s_{1}-1\right)+p_{1}^{3} \prod_{1}^{r^{2}} p_{l}^{2}\left(s_{2}-1\right)+q_{1}^{3} p_{1}^{3} \prod_{2}^{r^{3}} p_{l}^{3}\left(s_{3}-1\right)+\nu_{1}^{3}=0$ 
We consider the intersection of the planes (0), (1), (2) with $\left\{s_{3}=1\right\}$. It is easy to verify that the trace of the log-canonical wall is given by (1) and (2) and that the the three lines intersect. Then the first inequality does occur in the log-canonical wall.

(0) (1)

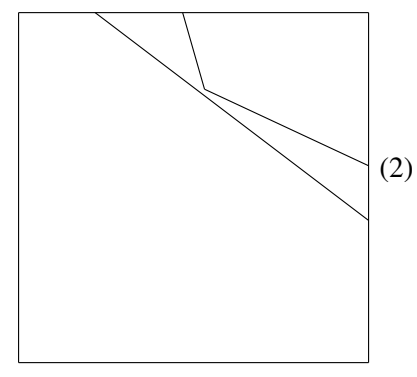

FiguRe 26

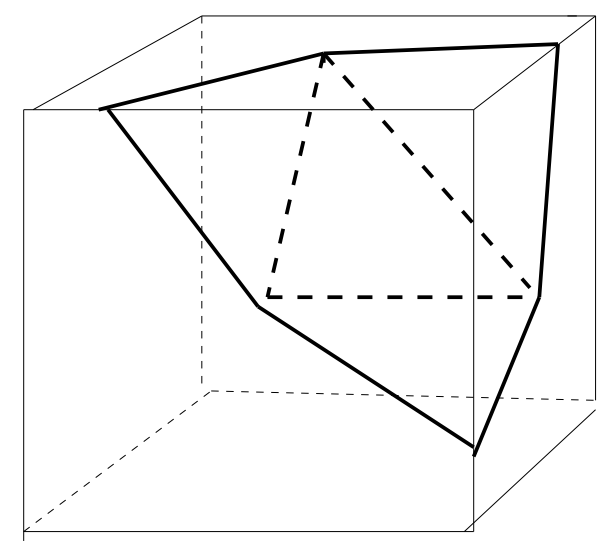

FiguRE 27

We have finished the proof of Theorem 4.1 (see Figure 27).

\subsection{Computation of the log-canonical wall.}

Definition 4.19. The Newton nest of a Newton tree is the set of vertices $v$ whose set of preceding vertices $\mathcal{S}_{v}$ is empty or satisfies, for all $v^{\prime} \in \mathcal{S}_{v}$ with nearby decorations $\left(q^{\prime}, p^{\prime}\right)$ either $p^{\prime}=1$ or $q^{\prime}=1$.

Remark 4.20. The Newton nest of a Newton tree doesn't depend on the system of coordinates since for all vertex in the Newton tree there exists a system of coordinates such that this vertex is on the Newton polygon. Note that there doesn't exist in general a system of coordinates such that all vertices are on the Newton polygon. 
EXAMPLE 4.21. (see figure 28)
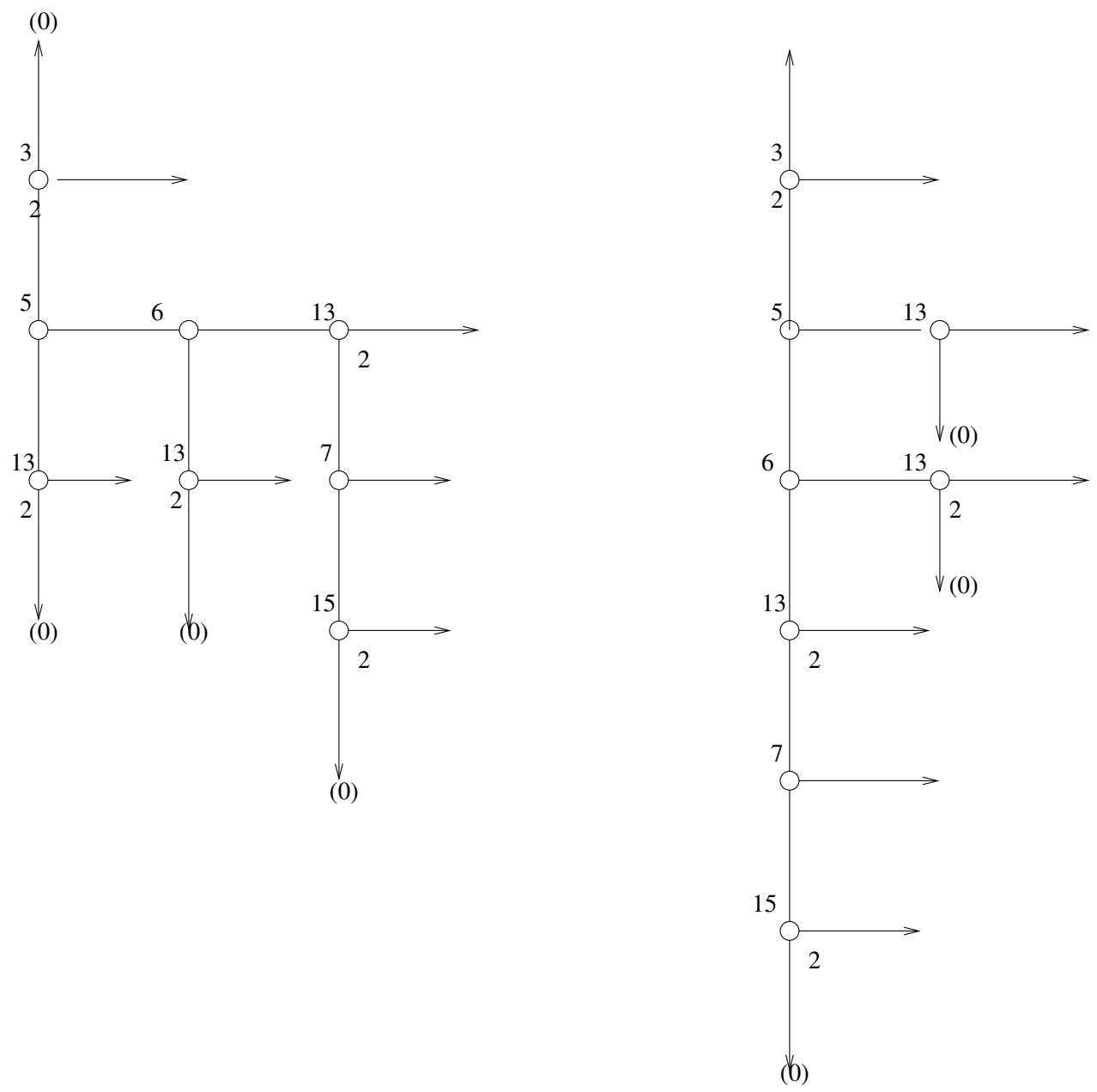

FiguRE 28

In this example all vertices of the Newton tree belong to the Newton nest of the Newton tree. On the right hand side of the figure we show the Newton tree in a different system of coordinates.

Theorem 4.22. (1) The log-canonical wall is given by the set of inequalities

$$
\sum N_{i, v}\left(s_{i}-1\right)+\nu_{v}>0
$$

where $v$ runs through the Newton nest of $f_{1} \cdots f_{r}$. 
(2) For each $v$ in the Newton nest of $f_{1} \cdots f_{r}$ there exists a face of the log canonical wall with equation

$$
\sum N_{i, v}\left(s_{i}-1\right)+\nu_{v}=0
$$

Corollary 4.23. The product of $\sum N_{i, v}\left(s_{i}-1\right)+\nu_{v}$ where $v$ runs over the Newton nest divides all polynomials in the Bernstein ideal.

The corollary is a consequence of Theorem 4.22 and [5].

Proof. The log-canonical wall is given by the inequalities

$$
\sum N_{i, v}\left(s_{i}-1\right)+\nu_{v}>0
$$

where $v$ runs through all the vertices of the Newton tree. We first prove that if $v$ doesn't belong to the Newton nest, then the corresponding inequality is implied by the inequalities with $v$ in the Newton nest. A vertex doesn't belong to the Newton nest if its set of preceding vertices $\mathcal{S}_{v}$ is not empty and if there exists $v^{\prime} \in \mathcal{S}_{v}$ with nearby decorations $\left(q^{\prime}, p^{\prime}\right)$ with $p^{\prime}>1$ and $q^{\prime}>1$. Let $v$ with nearby decorations $(q, p)$ be a vertex not in the Newton nest, $\left\{v_{0}, \cdots, v_{k}, v\right\}$ its set of preceding vertices. Let $j$ be the biggest index such that the nearby decorations of $v_{j}$ are $\left(q_{j}, p_{j}\right)$ with $p_{j}>1$ and $q_{j}>1$.

We have

$$
q=q_{k} p+\delta_{1}, q_{k}=q_{k-1}+\delta_{2}, \cdots q_{j+1}=q_{j} p_{j}+\delta_{k-j+1}
$$

Then $q=q_{j} p_{j} p+\delta_{1}+p\left(\delta_{2}+\cdots+\delta_{k-j+1}=q_{j} p_{j} p+\Delta\right.$.

$\nu_{v}=\nu_{v_{k}} p+\delta_{1}, \nu_{v_{k}}=\nu_{v_{k-1}}+\delta_{2}, \cdots, \nu_{v_{j-1}}=\nu_{v_{j}}+\delta_{k-j+1}, \nu_{v}=\nu_{v_{j}} p+\Delta$

We have

$$
\nu_{v} q_{j} p_{j}-\nu_{v_{j}} q=\Delta\left(p_{j} q_{j}-\nu_{j}\right)
$$

Consider $1 \leq i \leq r$. We have 3 cases: The intersection of the path between $v_{j}$ and the arrow representing $f_{i}$ and the path between $v_{j}$ and $v$ is

(1) empty

(2) is the path between $v_{j}$ and $v$

(3) is a non empty part of the path between $v_{j}$ and $v$

(1) In this case, we have $N_{i, v}=p N_{i, v_{j}}, \nu_{v}>\nu_{v_{j}} p$. Then $\frac{\nu_{v}}{N_{i, v}}>\frac{\nu_{v_{j}}}{N_{i, v_{j}}}$.

(2) In this case, we have $N_{i, v_{j}} q=p_{j} q_{j} N_{v}$ and since $p_{j}>1$ and $q_{j}>1, p_{j} q_{j}-\nu_{j}>0$ we deduce that $\frac{\nu_{v}}{N_{i, v}}>\frac{\nu_{v_{j}}}{N_{i, v_{j}}}$.

(3) We denote by $V$ the vertex where $f_{i}$ separates from the path between $v_{j}$ and $v$. We assume that $V$ has nearby decorations $(Q, P)$. We denote by $v_{l-1}$ the preceding vertex of $V$. We have $N_{i, v_{j}}=p_{j} q_{j} P N$ and $N_{i, v}=p Q N$. 


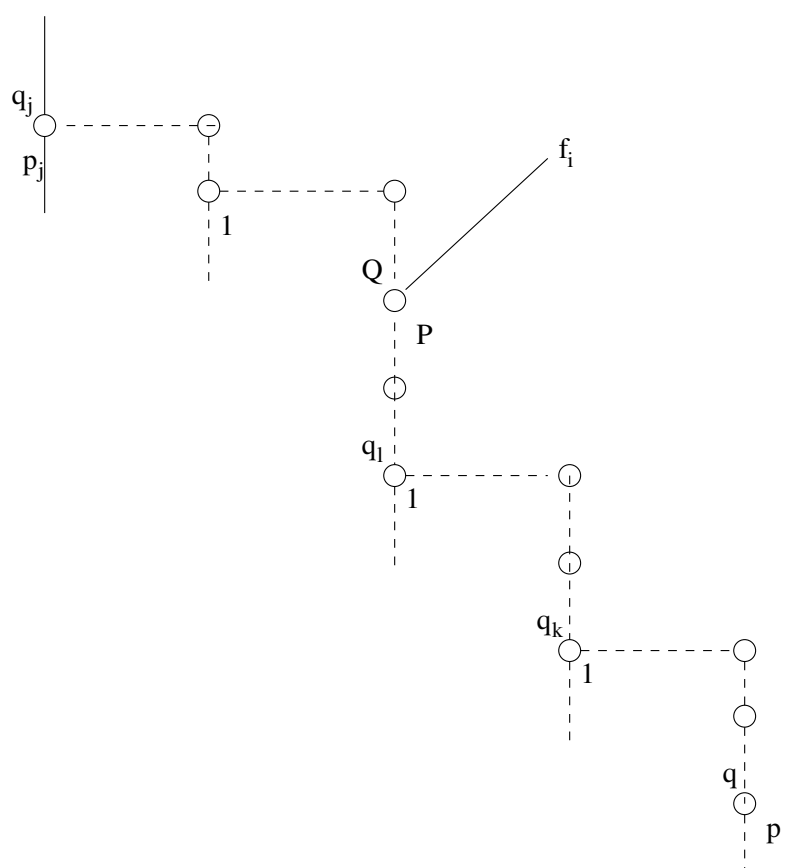

FiguRE 29

We have $q P-Q p>0$, then $p_{j} q_{j} N_{i, v}<q N_{i, v_{j}}$. Finally $\frac{\nu_{v}}{N_{i, v}}>\frac{\nu_{v_{j}}}{N_{i, v_{j}}}$. Then we have proved that the inequality for $v$ is implied by the inequality for $v_{j}$.

Now we have to prove that indeed all inequalities for $v$ in the Newton nest occur in the log-canonical wall. We use induction on $r$. The trace of the log-canonical wall on the hyperplane $\left\{s_{1}=1\right\}$ is the log-canonical wall of $f_{2}, \cdots, f_{r}$.

If $r=1$, the Newton nest consists in one vertex, the first one. Then the result is proved in this case. Assume that it is true for $r-1$. Consider $f_{1}, \cdots, f_{r}$. The Newton nest of $f_{1} \cdots f_{r}$ is the union of the Newton nests of $f_{1} \cdots f_{i-1} f_{i+1} \cdots f_{r}$ for all $i$ except in the exceptional cases. But we already proved that in these cases the log canonical wall is given by the Newton nest.

\section{EXAMPLE 4.24 .}

$$
f_{1}=y^{2}-x^{5}, \quad f_{2}=x^{4}-y^{3}
$$

The inequalities to be satisfied are

$$
10 s_{1}+8 s_{2}>11-(5 a+2 b) \quad\left(8 s_{1}+12 s_{2}>13-(4 a+3 b)\right.
$$

for all $(a, b) \in \mathbb{N}^{2}$. 


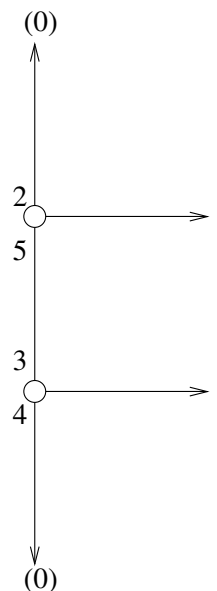

FiguRE 30

There are three pairs of half faces of quasi-adjunction given as the boundary of the sets

$$
\begin{gathered}
10 s_{1}+8 s_{2} \geq 11,8 s_{1}+12 s_{2} \geq 13 \\
10 s_{1}+8 s_{2} \geq 9,8 s_{1}+12 s_{2} \geq 10 \\
10 s_{1}+8 s_{2} \geq 7,8 s_{1}+12 s_{2} \geq 7
\end{gathered}
$$

The other faces of quasi-adjunction are:

$$
\begin{aligned}
& 10 s_{1}+8 s_{2}=5,10 s_{1}+8 s_{2}=3,10 s_{1}+8 s_{2}=1 \\
& 8 s_{1}+12 s_{2}=9,8 s_{1}+12 s_{2}=6,8 s_{1}+12 s_{2}=5,8 s_{1}+12 s_{2}=3,8 s_{1}+12 s_{2}=2,8 s_{1}+12 s_{2}=1 \\
& \quad \text { Any polynomial in the Bernstein ideal } \mathcal{B}_{f_{1}, f_{2}} \text { is divisible by } \\
& \prod_{i=3}^{i=8}\left(10 s_{1}+8 s_{2}+2 i+1\right)\left(8 s_{1}+12 s_{2}+7\right)\left(8 s_{1}+12 s_{2}+10\right)\left(8 s_{1}+12 s_{2}+11\right)\left(8 s_{1}+12 s_{2}+13\right) \\
& \left(8 s_{1}+12 s_{2}+14\right)\left(8 s_{1}+12 s_{2}+15\right)\left(8 s_{1}+12 s_{2}+17\right)\left(8 s_{1}+12 s_{2}+18\right)\left(8 s_{1}+12 s_{2}+19\right)
\end{aligned}
$$

EXAMPLE 4.25. The following example is degenerate.

$$
f_{1}=\left(x^{3}-y^{4}\right), \quad f_{2}=\left(\left(x^{2}-y^{3}\right)^{2}+x^{3} y^{2}\right)
$$

The log canonical wall is given by the two inequalities:

$$
12 s_{1}+8 s_{2}>1516 s_{1}+12 s_{2}>21
$$

There are two interesting features to notice in this example.

First we have a polytope of quasi-adjunction given by the three inequalities 


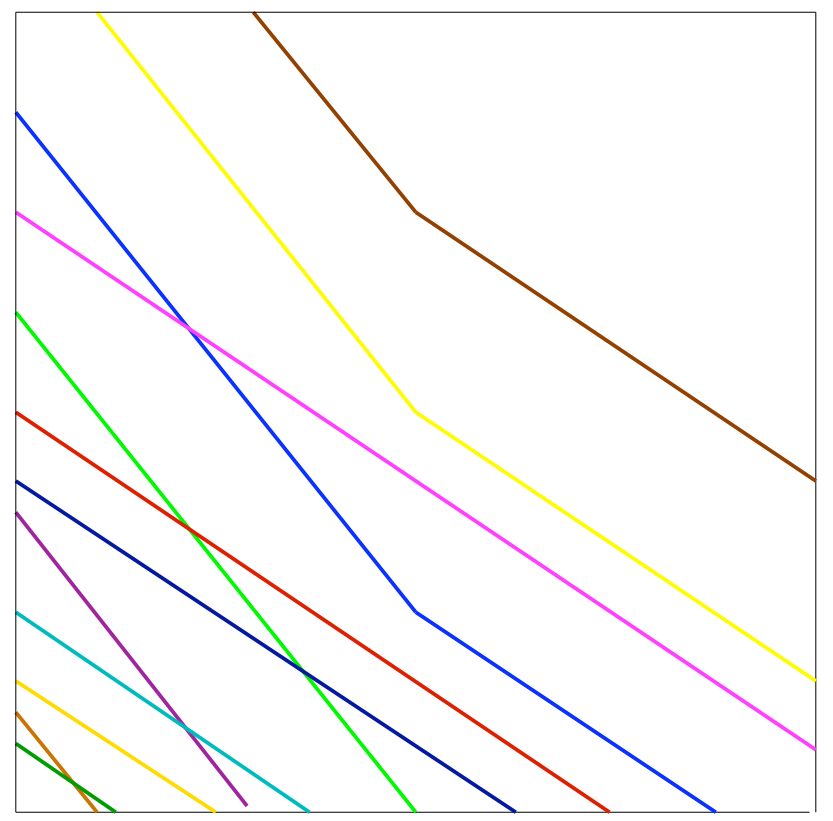

FIGURE 31

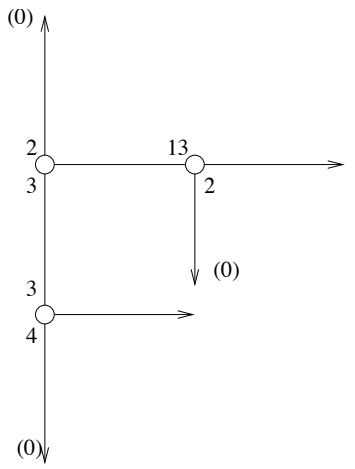

Figure 32

$$
12 s_{1}+8 s_{2}>1326 s_{1}+16 s_{2}>2716 s_{1}+12 s_{2}>18
$$

Second, we have a polytope of quasi-adjunction given by

$$
16 s_{1}+12 s_{2}>1726 s_{1}+16 s_{2}>25
$$

which shows that the set of vertices given a polytope of quasi-adjunction is not always connected in the Newton tree. 


\section{Distribution of CONStant AND Polytopes of QUASI-ADJUNCTION}

Shokurov made the conjecture and prove it in dimension two, that the set of log canonical thresholds satisfy the ACC condition. This means that there are no strictly increasing sequences of log canonical thresholds. More over it is proven also that the set of limits of strictly decreasing sequences of log canonical thresholds is the set $\{0,1 / n\}, n \in$ $\mathbb{N}$.

One can ask the question for other constant of quasi-adjunction. We can answer this question for the constants of quasi-adjunction associated to $\phi=x^{\alpha} y^{\beta}$.

Let $f$ be any germ in $\mathbb{C}[[x, y]]$. Consider its Newton tree, and denote by $\mathcal{V}$ the set of vertices of its Newton tree. Then the constant of quasiadjunction of $f$ associated to $\phi$ is

$$
\min _{v \in \mathcal{V}} \frac{\nu_{v}+e_{v}(\phi)}{N_{v}}
$$

If $\phi=1$, i.e. $\alpha=\beta=0$, it is the log canonical threshold.

First, we will give an example of a sequence of $f$ 's and $\phi$ for which the sequence of constants of quasi-adjunction is increasing.

EXAMPLE 5.1. Consider a germ with the following Newton tree.

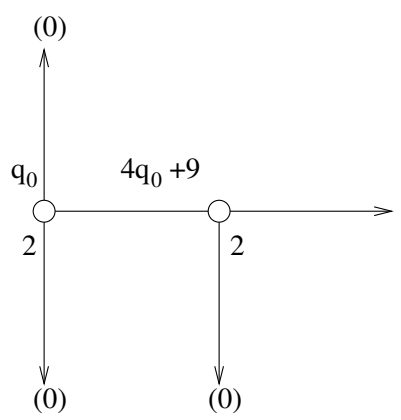

FiguRE 33

Let $\phi=y^{2}$. Then the associated constant of quasi-adjunction is

$$
\min \left\{\frac{2+3 q_{0}}{4 q_{0}}, \frac{13+6 q_{0}}{18+8 q_{0}}\right\}=\frac{13+6 q_{0}}{18+8 q_{0}}
$$

When $q_{0}$ goes to infinity $\frac{13+6 q_{0}}{18+8 q_{0}}$ is an increasing sequence which converges to $\frac{3}{4}$.

In [18], it is proven that the Ascending Chain Condition holds for LCT-polytopes, that is that all increasing chain of LCT-polytopes is 
eventually stationary. We give an example to show that it is no more true for the polytopes of quasi-adjunction associated to a $\phi \neq 1$.

EXAMPLE 5.2. We consider the germ with two irreducible components whose Newton tree is the following.

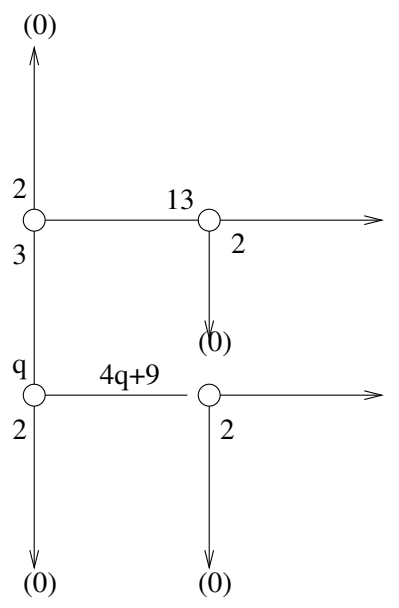

FiguRE 34

We take $\phi=y^{2}$. we have the four inequalities
(1) $12\left(s_{1}-1\right)+$
(2) $26\left(s_{1}-1\right)+$
$8\left(s_{2}-1\right)>$
$-9$
(3) $8\left(s_{1}-1\right)+$
$16\left(s_{2}-1\right)>$
$-19$
(4) $16\left(s_{1}-1\right)+$
$4 q\left(s_{2}-1\right)>$
$-(2+3 q)$
$2(4 q+9)\left(s_{2}-1\right)>-(13+6 q)$

The polytope of quasi-adjunction is given by the three lines
(1) $12\left(s_{1}-1\right)+$
$8\left(s_{2}-1\right)=$
$-9$
(2) $26\left(s_{1}-1\right)+$
$16\left(s_{2}-1\right)=$
$-19$
(4) $16\left(s_{1}-1\right)+2(4 q+9)\left(s_{2}-1\right)=-(13+6 q)$

When $q$ goes to infinity, the two first lines are fixed and the third one tends to the line $s_{2}=1 / 4$. Then we have a non stationary increasing sequence of polytopes.

Proposition 5.3. Consider a germ $f \in \mathbb{C}[[x, y]]$. Assume that the nearby decorations of the vertex connected by an edge to the upper arrow are $\left(q_{0}, p_{0}\right)$ and that $q_{0}$ goes to infinity. Then the constant of quasi-adjunction associated to $\phi=x^{\alpha} y^{\beta}$ tends to $\frac{\beta+1}{n}$, where $n$ is the multiplicity of $f$.

REMARK 5.4. We retrieve the result for the log canonical threshold that is when $\alpha=0, \beta=0$ the limit is $\frac{1}{n}$ proven by [12]. 


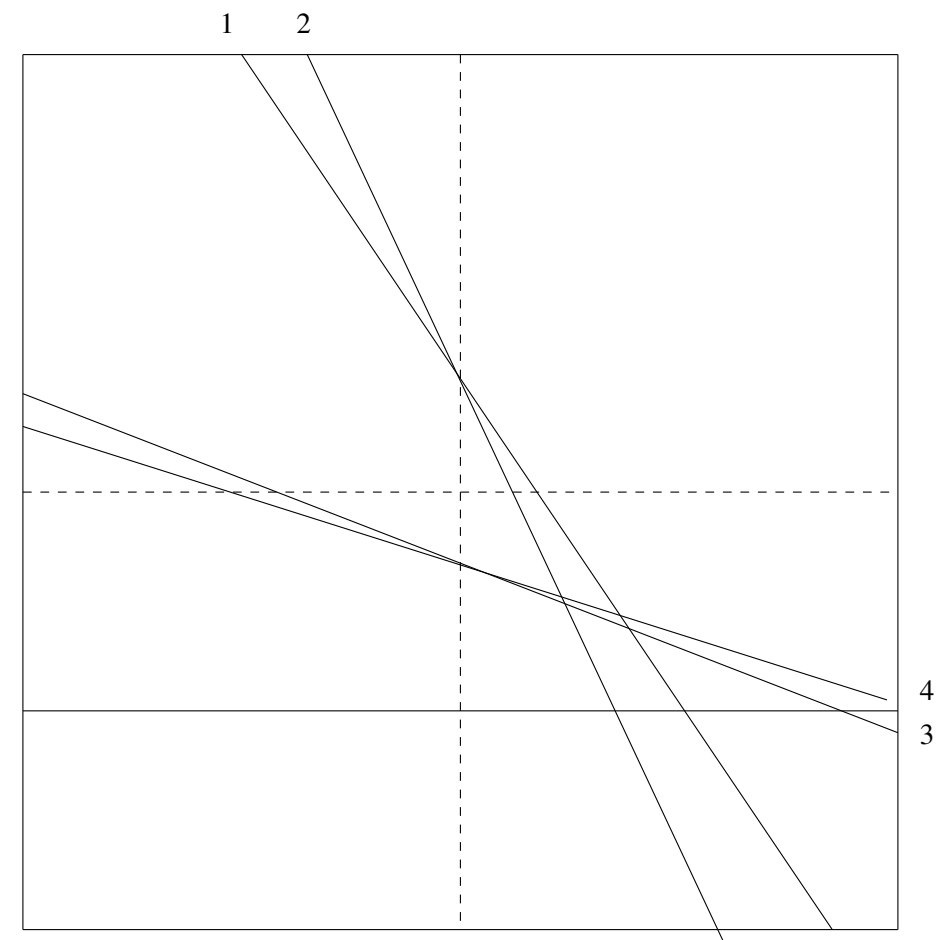

FiguRE 35

Proof. Let $\mathcal{V}$ be the set of vertices of the Newton tree. We have to consider

$$
\min _{\mathcal{V}} \frac{\nu_{v}+e_{v}(\phi)}{N_{v}}
$$

We will show that for all $v \in \mathcal{V}, \frac{\nu_{v}+e_{v}(\phi)}{N_{v}}$ tends to $\frac{\beta}{n}$ when $q_{0}$ goes to infinity.

We prove the result by induction on the number of successive vertical lines of the Newton tree.

Consider a vertex on the first vertical line.

For each vertex $v_{i}$ of the first vertical line, we define by $n_{i}$ the sum of the products of the numbers adjacent to the paths containing the horizontal edges issued from $v_{i}$, between $v_{i}$ and the arrows. We have

$$
n=\sum_{i} p_{j} n_{j}
$$

We have

$$
\nu_{i}+e_{i}(\phi)=(\alpha+1) p_{i}+(\beta+1) q_{i}
$$




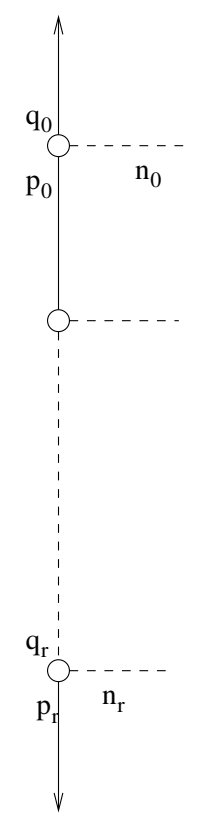

FiguRe 36

and

$$
N_{i}=q_{i} \sum_{i}^{r} p_{j} n_{j}+p_{i} \sum_{1}^{i-1} q_{j} n_{j}
$$

We denote by $\delta_{i}$ the edge determinant between $v_{i-1}$ and $v_{i}$. We can write

$$
q_{j}=q_{0} p_{j} / p_{0}+f_{j}(\delta, p)
$$

where $f_{j}$ depends only on the $\delta$ 's and $p$ 's. We have $q_{1} p_{0}-q_{0} p_{1}=\delta_{1}$. Then $q_{1}=q_{0} p_{1} / p_{0}+\delta_{1} / p_{0}$. Assume $q_{j}=q_{0} p_{j} / p_{0}+f_{j}(\delta, p)$. We have $q_{j+1} p_{j}-q_{j} p_{j+1}=\delta_{j+1}$, then $q_{j+1}=q_{0} p_{j+1} / p_{0}+f_{j}(\delta, p) p_{j+1} / p_{j}+\delta_{j+1} / p_{j}$, then $q_{j+1}=q_{0} p_{j+1} / p_{j}+f_{j+1}(\delta, p)$. We can write

$$
\begin{gathered}
N_{i}=q_{0} p_{i} / p_{0} \sum_{i}^{r} p_{j} n_{j}+p_{i} \sum_{1}^{i-1}\left(q_{0} p_{j} / p_{0}+f_{j}(\delta, p)\right) n_{j} \\
N_{i}=q_{0} p_{i} / p_{0} n+p_{i} \sum_{1}^{i-1} f_{j}(\delta, p) n_{j} \\
\nu_{i}+e_{i}(\phi)=(\alpha+1) p_{i}+(\beta+1)\left(q_{0} p_{i} / p_{0}+f_{i}(\delta, p)\right.
\end{gathered}
$$

Then the assertion is proved for every vertex on the first vertical line.

We assume now that the assertion is true for all the vertices of the $k$-th vertical line. 

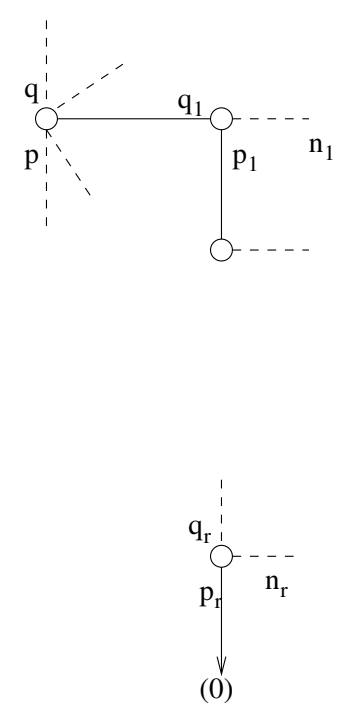

FiguRE 37

We consider a vertex $v$ on the $k$-th vertical line and we assume that for this vertex

$$
\lim _{q_{0} \rightarrow \infty} \frac{\nu+e(\phi)}{N}=\frac{\beta+1}{n}
$$

Consider a vertex $v_{i}$ on a $k+1$-th vertical line issued from $v$. We have

$$
\nu_{i}+e_{i}(\phi)=\nu p_{i}+\delta_{i}^{\prime}+e(\phi) p_{i}
$$

where $\delta_{i}^{\prime}=q_{i}-q p p_{i}$. We can write

$$
N=N^{\prime}+p q \sum_{1}^{r} p_{j} n_{j}
$$

Then

$$
\begin{gathered}
N_{i}=N^{\prime} p_{i}+p_{i} \sum_{1}^{i-1} q_{j} n_{j}+q_{i} \sum_{i}^{r} p_{j} n_{j} \\
N_{i}=N^{\prime} p_{i}+p_{i} \sum_{1}^{i-1}\left(q p p_{j}+\delta_{j}^{\prime}\right) n_{j}+\left(q p p_{i}+\delta_{i}^{\prime}\right) \sum_{i}^{r} p_{j} n_{j} \\
N_{i}=p_{i} N+p_{i} \sum_{1}^{i-1} \delta_{j}^{\prime} n_{j}+\delta_{i}^{\prime} \sum_{i}^{r} p_{j} n_{j}
\end{gathered}
$$

Then

$$
\frac{\nu_{i}+e_{i}(\phi)}{N_{i}}=\frac{(\nu+e(\phi)) p_{i}+\delta_{i}^{\prime}}{p_{i} N+p_{i} \sum_{54}^{i-1} \delta_{j}^{\prime} n_{j}+\delta_{i}^{\prime} \sum_{i}^{r} p_{j} n_{j}}
$$


Then the result is proved.

\section{REFERENCES}

[1] A.Adem, J.Leida, Y. Ruan, Orbifolds and stringy topology. Cambridge Tracts in Mathematics, 171. Cambridge University Press, Cambridge, 2007.

[2] E. Artal Bartolo, P. Cassou-Noguès, I. Luengo, A. Melle HERNÁNDEZ, On $\nu$-quasi-ordinary power series: factorization, Newton trees and resultants. Topology of algebraic varieties and singularities, 321343, Contemp. Math., 538, Amer. Math. Soc., Providence, RI, 2011

[3] N.A'CAmpo, M.OKA, Geometry of plane curves via Tschirnhausen resolution tower. Osaka J. Math. 33 (1996), no. 4, 1003-1033.

[4] P. CAssou-Noguès, Newton trees at infinity of algebraic curves. Affine algebraic geometry, 119, CRM Proc. Lecture Notes, 54, Amer. Math. Soc., Providence, RI, 2011.

[5] P. Cassou-Noguès, A. Libgober, Multivariable Hodge theorical invariants of germs of plane curves. Journal of Knot theory and its ramifications, vol. 20, n $6,(2011), 787-805$.

[6] P. Cassou-Noguès, W. Veys, Newton trees for ideals in two variables and applications. ArXiv 1201.0467.

[7] P. Cassou-Noguès, W.Veys, In preparation.

[8] D. Eisenbud, W. Neumann, Three dimensional link theory and invariants of plane curve singularities, Annals of Mathematic studies, 110, Princeton University press, Princeton, NJ, (1985).

[9] W.Fulton, Introduction to toric varieties. Annals of Mathematics Studies, 131. The William H. Roever Lectures in Geometry. Princeton University Press, Princeton, NJ, 1993.

[10] P.D Gonzalez PÉrez, Toric embedded resolutions of quasi-ordinary hypersurface singularities. Ann. Inst. Fourier (Grenoble) 53 (2003), no. 6, 18191881.

[11] A.GyouA, Bernstein-Sato's polynomial for several analytic functions. J. Math. Kyoto Univ. 33 (1993), no. 2, 399-411.

[12] J.Kollar, Singularities of pairs. Algebraic geometry - Santa Cruz 1995, 221287, Proc. Sympos. Pure Math., 62, Part 1, Amer. Math. Soc., Providence, RI, 1997.

[13] Y.Kawamata, On algebraic fiber spaces. Contemporary trends in algebraic geometry and algebraic topology (Tianjin, 2000), 135154, Nankai Tracts Math., 5, World Sci. Publ., River Edge, NJ, 2002.

[14] G.Kempf, F.Knudsen, D.Mumford, B.Saint-Donat, Toroidal embeddings. I. Lecture Notes in Mathematics, Vol. 339. Springer-Verlag, Berlin-New York, 1973.

[15] D.T.LÊ, M.OkA, On resolution complexity of plane curves. Kodai Math.J. 18 (1995), 1-36.

[16] A.Libgober, Characteristic varieties of algebraic curves. Applications of algebraic geometry to coding theory, physics and computation (Eilat, 2001), 215-254, NATO Sci. Ser. II Math. Phys. Chem., 36, Kluwer Acad. Publ., Dordrecht, 2001.

[17] A.Libgober, Hodge decomposition of Alexander invariants. Manuscripta Math. 107 (2002), no. 2, 251-269. 
[18] A. Libgober, M. Mustata, Sequences of LCT-polytopes. Math. Res. Lett. 18 (2011), no. 4, 733746.

[19] F.Loeser, M.Vaquie, Le polynme d'Alexander d'une courbe plane projective. (French) [The Alexander polynomial of a projective plane curve] Topology 29 (1990), no. 2, 163173

[20] A. Nemethi, W. Veys, Generalized monodromy conjecture in dimension two. ArXiv 1112.1230.

[21] M. OKA, Geometry of plane curves via toroidal resolution. Algebraic geometry and Singularities, (La Rabida, 1991) 95-121, Prog. Math, 134, Birkhauser, basel, 1996.

[22] C.Sabbah, Proximité évanescente. I. La structure polaire d'un $D$-module. Compositio Math. 62 (1987), no. 3, 283-328, Proximité évanescente. II. Equations fonctionnelles pour plusieurs fonctions analytiques. Compositio Math. 64 (1987), no. 2, 213-241.

[23] M.Saito, Exponents of an irreducible plane singularity. ArXiv 0009133.

[24] B.Totaro, The ACC conjecture for log canonical thresholds (after de Fernex, Ein, Mustata, Kollar), Asterisque (2011), no. 339, Exp. No. 1025, ix, 371385, Seminaire Bourbaki. Vol. 2009/2010. Expose 10121026.

[25] C.T.C.WALL, Singular points of plane curves. London Mathematical Society Student Texts, 63. Cambridge University Press, Cambridge, 2004.

[26] J.WlodarczyK, Toroidal varieties and the weak factorization theorem. Invent. Math. 154 (2003), no. 2, 223331.

[27] W. Veys, Zeta functions for curves and log canonical models. Proc. London Math. Soc. (3) 74 (1997), no. 2, 360378.

Institut de mathématiques de Bordeaux,, Université Bordeaux I, 351, Cours de la Libération, 33405, Talence Cedex, France.

E-mail address: Pierrette.Cassou-nogues@math.u-bordeaux1.fr

Department of Mathematics, University of Illinois, Chicago, IL 60607

E-mail address: libgober@math.uic.edu 